\section{Revista de CIENCIAS AMBIENTALES Tropical Journal of Environmental Sciences}

Revista de Ciencias Ambientales (Trop J Environ Sci) e-ISSN: 2215-3896

(Julio-Diciembre, 2021) . Vol 55(2): 19-44 DOI: https://doi.org/10.15359/rca.55-2.2

Open Access: www.revistas.una.ac.cr/ambientales e-mail: revista.ambientales@una.ac.cr Kubota V., Pulleman M., Domínguez A., Montiel C., Pineda Y., Ortiz A., Kubsch N., Salas D. y Galeano P.

\title{
Efectos de diferentes sistemas de uso de suelo sobre la diversidad de árboles, almacenamiento de carbono y calidad del suelo en el Bosque Atlántico del Alto Paraná, Paraguay
}

\author{
Effects of different land use systems on tree diversity, carbon stocks and soil quality \\ in the Upper Parana Atlantic Forest, Paraguay
}

\begin{abstract}
Victoria Rika Kubota ${ }^{1,2}$, Mirjam M. Pulleman ${ }^{3,4}$, Aaron Domínguez ${ }^{5}$, Carmen Montiel ${ }^{6}$, Yomali Pineda ${ }^{7}$, Andrea Ortiz ${ }^{8}$, Nadine Kubsch ${ }^{9}$, Danilo Salas ${ }^{10}$, Pilar Galeano ${ }^{11}$
\end{abstract}

[Recibido: 27 de julio 2020, Aceptado: 28 de enero 2021, Corregido: 17 de marzo 2021, Publicado: 1 de julio 2021]

\section{Resumen}

[Introducción]: La evaluación del suministro de servicios ecosistémicos por los diferentes usos del suelo de la agricultura familiar facilita plantear estrategias para el uso sostenible de la tierra. [Objetivo]: Se determinaron los efectos de diferentes usos del suelo practicados en la agricultura familiar, sobre la conservación de la diversidad arbórea y el suministro de servicios ecosistémicos críticos: almacenamiento de carbono aéreo y subterráneo y servicios de soporte que provienen del suelo, en la Reserva de Biosfera del Bosque Mbaracayú (Canindeyú, Paraguay). [Metodología]: Fue determinada la biodiversidad arbórea, el almacenamiento de carbono aéreo y de suelo, las propiedades químicas y físicas del suelo en 7 fincas con diversos tipos de cobertura: bosque nativo alterado, sistemas agroforestales con yerba mate, cultivo anual de subsistencia y pasturas. En cada uno de los puntos de muestreo, fueron registrados datos de los individuos arbóreos y arbustivos, y fueron tomadas muestras del estrato herbáceo, hojarasca y suelo. [Resultados]: Se registró mayor índice de diversidad y almacenamiento de carbono aéreo en bosques nativos alterados y sistemas agroforestales, sin diferencia significativa entre ellos. Los valores de

1 Técnico de la Facultad de Ciencias Agrarias, Universidad Nacional de Asunción, Paraguay, kubotapy@agr.una.py, https://orcid. org/0000-0001-7080-7406

2 Técnico de la División de Áreas Protegidas, Dirección de Coordinación Ejecutiva, ITAIPU Binacional, Paraguay, kubota@itaipu.gov.py, https://orcid.org/0000-0001-7080-7406

3 Investigador del Centro Internacional de Agricultura Tropical (CIAT), Colombia, m.pulleman@cgiar.org, https://orcid. org/0000-0001-9950-0176

4 Investigador de Soil Biology Group, Wageningen University, Países Bajos, mirjam.pulleman@wur.nl, https://orcid.org/0000-0001-9950-0176

5 Egresado de la Facultad de Ciencias Agrarias, Universidad Nacional de Asunción, Paraguay, aaron.dominguez.melgarejo@gmail.com, https://orcid.org/0000-0003-2146-6929

6 Egresado de la Facultad de Ciencias Agrarias, Universidad Nacional de Asunción, Paraguay, kim.montiel@gmail, https://orcid. org/0000-0003-4869-7057

7 Egresado de la Facultad de Ciencias Agrarias, Universidad Nacional de Asunción, Paraguay, yomali.pineda@gmail.com, https://orcid. org/0000-0001-6528-484x

8 Estudiante de la Facultad de Ciencias Agrarias, Universidad Nacional de Asunción, Paraguay, eaov37@gmail.com, https://orcid. org/0000-0002-8347-7220

9 Investigador del Centro Internacional de Agricultura Tropical (CIAT), Colombia, nkubsch@posteo.de, https://orcid. org/0000-0002-6308-8877

10 Investigador de la Fundación Moisés Bertoni, Paraguay, dsalas@mbertoni.org.py, https://orcid.org/0000-0002-3242-6052

11 Docente de la Facultad de Ciencias Agrarias, Universidad Nacional de Asunción, Paraguay, pilar.galeano68@gmail.com, https://orcid. org/0000-0002-0621-8639

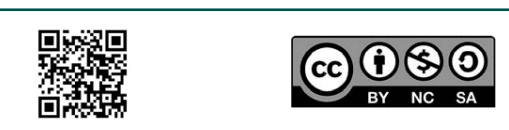

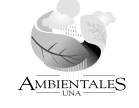




\section{Revista de CIENCIAS AMBIENTALES Tropical Journal of Environmental Sciences}

Revista de Ciencias Ambientales (Trop J Environ Sci) e-ISSN: 2215-3896

(Julio-Diciembre, 2021) . Vol 55(2): 19-44 DOI: https://doi.org/10.15359/rca.55-2.2

Open Access: www.revistas.una.ac.cr/ambientales e-mail: revista.ambientales@una.ac.cr Kubota V., Pulleman M., Domínguez A., Montiel C., Pineda Y., Ortiz A., Kubsch N., Salas D. y Galeano P.

CIC y $\mathrm{Ca}^{+2}$ de suelo fueron más elevados en los bosques nativos alterados comparado con pasturas, y los otros usos presentaron valores intermedios. No hubo diferencias significativas entre los diferentes usos en cuanto al carbono almacenado ni en estabilidad de la estructura del suelo. Sin embargo, las pasturas presentaron índices de calidad de suelo más bajos. [Conclusiones]: Se demostró que bosques nativos alterados y sistemas agroforestales aportan más a la conservación de la diversidad arbórea, retención de carbono y calidad del suelo.

Palabras clave: Agricultura familiar; bosque nativo alterado; sistemas agroforestales con yerba mate; Reserva de Biosfera del Bosque Mbaracayú; servicios ecosistémicos.

\begin{abstract}
[Introduction]: The evaluation of ecosystem services in different land use systems in family farming facilitates the development of sustainable land use strategies. [Objective]: We studied the effects of different land uses, commonly applied in family farming in the Mbaracayú Forest Biosphere Reserve (Canindeyú, Paraguay), on the conservation of tree diversity and delivery of critical ecosystem services: above and belowground carbon storage and supporting services rendered by soil. [Methods]: We determined the diversity of trees, the stocks of carbon above ground and in the soil, as well as chemical and physical soil properties in 7 farms with diverse land use systems: disturbed native forest, agroforestry system with yerba mate, subsistence crops and pastures. In each of the sampling points, data of the tree and shrub individuals were recorded, and samples of the herbaceous stratum, litter and soil were taken. [Results]: We found the highest rate of both diversity and above ground carbon storage in the disturbed native forests and agroforestry systems without significant difference between the two. The values of CEC and $\mathrm{Ca}^{+2}$ in the soil were higher in disturbed native forests compared to pastures, while the remaining land uses showed intermediate values. There was no significant difference between the different land use types regarding carbon storage and physical stability of the soils. Nonetheless, the pastures had the lowest soil qualities. [Conclusions]: We demonstrated that the altered native forests and agroforest systems with yerba mate promote better the conservation of tree diversity, carbon retention and soil quality.
\end{abstract}

Keywords: disturbed native forest; ecosystem services; family farming; yerba mate agroforestry; Mbaracayú Forest Biosphere Reserve.

\title{
1. Introducción
}

Existe un aumento de presión sobre los recursos naturales, ocasionando deforestación, fragmentación y degradación de diversos ecosistemas a nivel mundial, incluyendo los hotspots de biodiversidad (Weinzettel et al., 2018). Ante esto, urge buscar estrategias para conciliar la conservación de recursos naturales y los objetivos del desarrollo. La agricultura familiar depende de los servicios ecosistémicos para su subsistencia, pero a la vez aporta a la conservación de biodiversidad y a la provisión de distintos servicios, siendo algunos sistemas de uso del suelo más beneficiosos que otros (Grossman, 2015). Considerando los aspectos sociales, ambientales y económicos de la agricultura familiar, es relevante cuantificar y documentar la relación entre los diversos sistemas de uso del suelo y los servicios ecosistémicos.

Un caso en específico que demuestra esta problemática es el Bosque Atlántico del Alto Paraná (BAAPA), compartido por Brasil, Paraguay y Argentina con una superficie de 47120400 ha (Di Bitetti et al., 2003) y originalmente ocupado por bosques subtropicales semideciduos con la estructura más compleja y con mayor contenido de biomasa de Paraguay (Tortorelli, 1967).

\begin{tabular}{|c|c|c|}
\hline 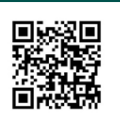 & (c) (i) (\$) & 20 \\
\hline
\end{tabular}




\section{Revista de CIENCIAS AMBIENTALES Tropical Journal of Environmental Sciences}

Revista de Ciencias Ambientales (Trop J Environ Sci) e-ISSN: 2215-3896

(Julio-Diciembre, 2021) . Vol 55(2): 19-44 DOI: https://doi.org/10.15359/rca.55-2.2

Open Access: www.revistas.una.ac.cr/ambientales e-mail: revista.ambientales@una.ac.cr Kubota V., Pulleman M., Domínguez A., Montiel C., Pineda Y., Ortiz A., Kubsch N., Salas D. y Galeano P.

Además, es una ecorregión muy rica en biodiversidad, siendo uno de los hotspots de biodiversidad (Cartes, 2005). En décadas recientes, extensas superficies del BAAPA fueron deforestadas $\mathrm{y}$ transformadas principalmente en monocultivos empresariales como soja y pasturas (De Sy et al., 2015). En Paraguay, la cobertura forestal del BAAPA se redujo al 73 \% en 1973, al $24.9 \%$ en 2000 (Huang et al., 2007), generando efectos negativos sobre la biodiversidad, propiedades de suelo y la oferta de servicios ecosistémicos (De Sy et al., 2015; De Souza et al., 2012).

La Reserva de Biosfera del Bosque Mbaracayú (RBBM), de 291962 ha, se encuentra en la ecorregión BAAPA y desde el año 2000 es reconocida como tal por la Organización de las Naciones Unidas para la Educación, la Ciencia y la Cultura (UNESCO). Presenta un paisaje biofísicamente heterogéneo, formado por un mosaico de usos del suelo, y en su territorio se busca la conciliación entre el bienestar socioeconómico y la conservación de la naturaleza. En el año 2001, aproximadamente 192115 ha de la superficie de esta reserva de biosfera correspondían a diversos tipos de vegetación natural, 49037 ha a uso pecuario, 42709 ha a la agricultura familiar (fincas con $<20$ ha) y 8102 ha a la agricultura empresarial (Carlson et al., 2011). En el año 2015, 148204.82 ha de la superficie de esta reserva correspondían a la cobertura boscosa, de las cuales 57183 ha pertenecían a la Reserva Natural del Bosque Mbaracayú (Huang et al., 2007). La ecorregión BAAPA se encuentra cada vez más afectada por la expansión de la agricultura empresarial, como es el caso en la zona de la RBBM (Ministerio de Agricultura y Ganadería [MAG], 2009).

La agricultura familiar en la zona se caracteriza por una alta diversidad de cultivos de subsistencia, ganado, agroforestales, silvopastoriles, y pequeñas áreas con remanentes de bosques intervenidos, formando un mosaico de paisajes con distintos usos de suelo (Grossman, 2015). Este tipo de agricultura se caracteriza por estar asentada en suelos de baja fertilidad, con bajos niveles de aplicación de tecnología, escasa asistencia técnica y crediticia y baja capacidad para la comercialización de sus productos (Zarza, 2017). En estas fincas se produce una variedad de cultivos de subsistencia como mandioca (Manihot esculenta), poroto (Phaseolus vulgaris) y maní (Arachis hypogaea), cultivos comerciales como algodón (Gossypium sp.), maíz (Zea mays), soja (Glycine max), sésamo (Sesamum indicum), plantación de yerba mate (Ilex paraguariensis) o Citrus sp., pastura para la ganadería y eventualmente plantaciones forestales de especies introducidas (Carlson et al., 2011; MAG, 2009).

La relevancia de los bosques remanentes en la RBBM sobre la protección de acuíferos, mediante la oferta de mayor provisión de servicios ecosistémicos de amortiguación de inundaciones, disponibilidad de agua subterránea y superficial, fue reportada por Galluppi-Selich et al. (2019). Considerando el papel multifuncional de la vegetación, es relevante analizar y cuantificar la oferta de los diversos servicios ecosistémicos que ofrecen los distintos usos del suelo de la agricultura familiar que integran el mosaico de paisaje de la región (Grossman, 2015) con el fin de poder plantear estrategias que permitan el uso sostenible y eficiente del suelo en estos paisajes.

\begin{tabular}{|c|c|c|c|c|c|}
\hline 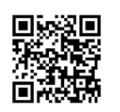 & (c) () () () & $\underset{\text { AMBEIETILES }}{8}$ & $\frac{1 \%}{2 \%}$ & 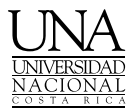 & 21 \\
\hline
\end{tabular}




\section{Revista de CIENCIAS AMBIENTALES Tropical Journal of Environmental Sciences}

Revista de Ciencias Ambientales (Trop J Environ Sci) e-ISSN: 2215-3896

(Julio-Diciembre, 2021) . Vol 55(2): 19-44 DOI: https://doi.org/10.15359/rca.55-2.2

Open Access: www.revistas.una.ac.cr/ambientales e-mail: revista.ambientales@una.ac.cr Kubota V., Pulleman M., Domínguez A., Montiel C., Pineda Y., Ortiz A., Kubsch N., Salas D. y Galeano P.

El objetivo del estudio fue determinar los efectos de los diferentes usos del suelo de la agricultura familiar practicada en la RBBM, sobre la conservación de la diversidad de especies arbóreas y el suministro de los servicios ecosistémicos, enfocado en el almacenamiento de carbono en la biomasa aérea, en la hojarasca, en el suelo, y en los servicios de soporte que provienen del suelo tales como: disponibilidad de nutrientes, estructura, calidad química y física del suelo.

\section{Metodología}

\section{1 Área de estudio}

El estudio fue desarrollado en la RBBM, Departamento Canindeyú, Paraguay (Figura 1). El clima es típicamente continental, clasificando según Koeppen como templado lluvioso (Cfa) y según el sistema de Holdridge como zona de vida de bosque húmedo templado cálido (BhTc) (FMB, 2005). La precipitación media es de 1600 a $1700 \mathrm{~mm}$ y la temperatura es de 21 a $22{ }^{\circ} \mathrm{C}$ (FMB, 2005). El paisaje es de tipo lomada y el suelo dominante es el Arenic Rhodic Paleudult Ultisol (López-Gorostiaga et al., 1998).

El estudio se enfocó en los usos del suelo practicados en la agricultura familiar dentro de la RBBM, adoptando la definición propuesta por la Sección Nacional Paraguaya de la Reunión Especializada en Agricultura Familiar (REAF), que indica que la agricultura familiar en la región Oriental de Paraguay utiliza principalmente fuerza de trabajo familiar para hacer producir el predio, que estos miembros de la familia residen en la finca y/o comunidades cercanas y que no utiliza más de 50 ha (Zarza, 2017).

Los datos fueron recopilados en fincas distribuidas en 4 comunidades de la RBBM: Tendal, 11 de Setiembre, Arroyo Guazú y Las Residentas. Fueron elegidas 7 fincas con una historia de uso del suelo similar y que corresponde a fincas modelos de la Fundación Moisés Bertoni, organización encargada de la gestión de la RBBM (Apéndice 1). En la zona de estudio no hay variabilidad topográfica.

Fueron considerados 4 tipos de uso del suelo presentes en cada una de las fincas seleccionadas: bosque nativo alterado, sistema agroforestal con yerba mate, cultivo anual de subsistencia (mandioca) y pasturas. Los bosques nativos alterados fueron pequeños fragmentos de remanentes boscosos o bosques en regeneración dentro de las fincas que sirven de fuente de madera y leña para los residentes. Las plantas de yerba mate tenían 1 a 2 años en el momento de la toma de datos. La cobertura protectora del sistema agroforestal fueron bosques alterados en algunos casos y arbustales en otros. Las pasturas compuestas por diversas especies de gramíneas y herbáceas estaban destinadas a la cría de ganado bovino. Además, se hizo un muestreo en 4 parcelas adicionales dentro del mismo paisaje con cultivos comerciales en donde se había cultivado soja al menos en los 2 últimos años, totalizando 32 puntos de muestreo. Según datos brindados por los propietarios, en las fincas con plantaciones comerciales (soja y maíz) con labranza mecanizada se practica la rotación de cultivos y además se aplican pesticidas y fertilizantes.

\begin{tabular}{|c|c|c|c|c|c|}
\hline 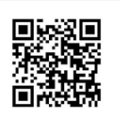 & (c) (i) $\$(0)$ & $\underbrace{}_{\text {AMBIFNAIIES }}$ & $\begin{array}{l}\frac{9 \%}{2} \\
\frac{2}{2} \\
\text { euna }\end{array}$ & 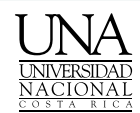 & 22 \\
\hline
\end{tabular}




\section{Revista de CIENCIAS AMBIENTALES Tropical Journal of Environmental Sciences}

Revista de Ciencias Ambientales (Trop J Environ Sci) e-ISSN: $2215-3896$

(Julio-Diciembre, 2021) . Vol 55(2): 19-44 DOI: https://doi.org/10.15359/rca.55-2.2 Open Access: www.revistas.una.ac.cr/ambientales e-mail: revista.ambientales@una.ac.cr Kubota V., Pulleman M., Domínguez A., Montiel C., Pineda Y., Ortiz A., Kubsch N., Salas D. y Galeano P.

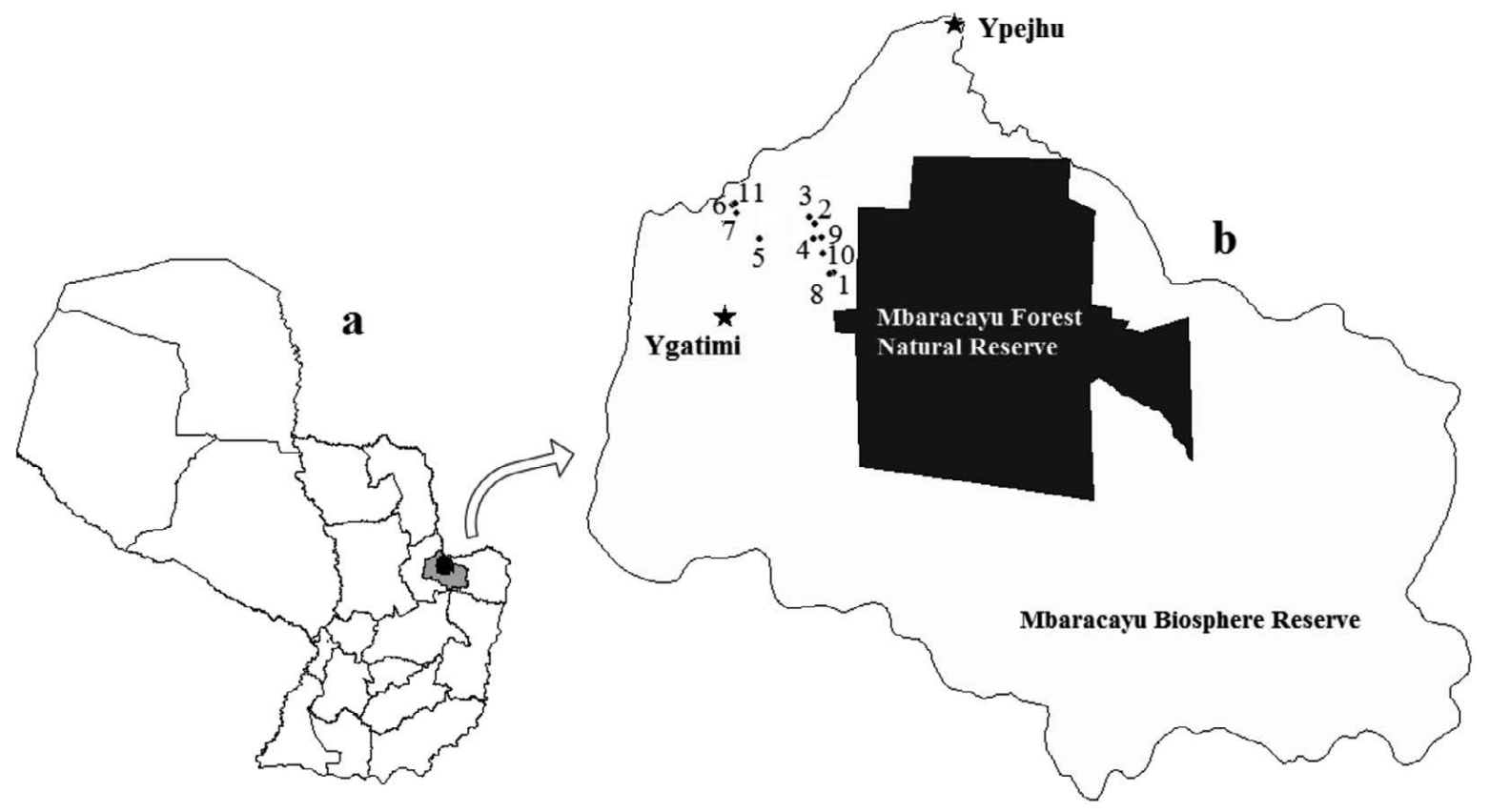

Figura 1. Ubicación del área de estudio. a. Ubicación de la Reserva de Biosfera del Bosque Mbaracayú (polígono gris) y la Reserva Natural de Bosque Mbaracayú (polígono negro) en Paraguay. b. Ubicación de las fincas dentro de la Reserva de Biosfera del Bosque Mbaracayú (números 1-11).

Figure 1. Location of the study sites. a. Location of Mbaracayú Forest Biosphere Reserve (gray polygon) and Mbaracayú Forest Natural Reserve (black polygon) in Paraguay, b. Location of the farms in Mbaracayú Forest Biosphere Reserve (numbers 1-11).

\subsection{Registro de datos y toma de muestras de vegetación}

Fue instalada una parcela temporal de 0.1 ha $(20 \times 50 \mathrm{~m})$ (Figura 2), en cada uno de los 32 puntos para el muestreo del estrato arbóreo incluyendo Guadua aff. chacoensis y palmeras, estrato arbustivo incluyendo la mandioca, estrato herbáceo y la hojarasca.

En el compartimiento de $20 \times 50 \mathrm{~m}$ fueron registrados datos de especie y DAP (diámetro a la altura de pecho, a $1.3 \mathrm{~m}$ desde el suelo) de los individuos con DAP $\geq 10 \mathrm{~cm}$ y datos de especie, DAP y Hstem (altura del estípite) para las palmeras. La identificación de las especies estuvo a cargo de un asistente de campo, oriundo de la zona.

En las 3 subparcelas (A1, A2 y A3) de 0.005 ha $(5 \times 10 \mathrm{~m})$ establecidas dentro de la parcela de 0.1 ha, se registraron datos de DAP de los individuos arbustivos con DAP entre 2 y $9.9 \mathrm{~cm}$, y con altura igual o superior a $1.5 \mathrm{~m}$ (Figura 2). Todos los individuos de mandioca fueron contabilizados y se seleccionaron aleatoriamente 10 plantas dentro de cada subparcela (30 plantas por punto de muestreo), para registrar su DAP y altura.

El estrato herbáceo (vegetación con DAP $<2 \mathrm{~cm}$ ) y la hojarasca (hojas, flores, frutos, semillas y ramas con diámetro menor a $2 \mathrm{~cm}$ depositados sobre el suelo) fueron muestreados en 5

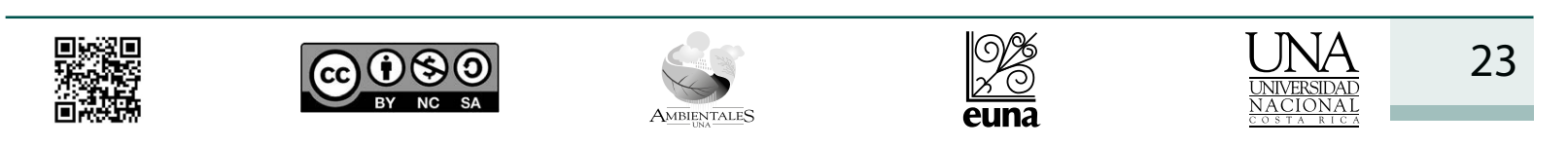




\section{Revista de CIENCIAS AMBIENTALES Tropical Journal of Environmental Sciences}

Revista de Ciencias Ambientales (Trop J Environ Sci) e-ISSN: 2215-3896

(Julio-Diciembre, 2021) . Vol 55(2): 19-44 DOI: https://doi.org/10.15359/rca.55-2.2 Open Access: www.revistas.una.ac.cr/ambientales e-mail: revista.ambientales@una.ac.cr Kubota V., Pulleman M., Domínguez A., Montiel C., Pineda Y., Ortiz A., Kubsch N., Salas D. y Galeano P.

puntos equidistantes ubicados sobre la línea diagonal que atraviesa la parcela de $0.1 \mathrm{ha}(\mathrm{H} 1, \mathrm{H} 2$, $\mathrm{H} 3, \mathrm{H} 4$ y H5, Figura 2). Fueron empleados bastidores de $50 \times 50 \mathrm{~cm}$ para el muestreo de ambos estratos. La parte aérea de las hierbas fue cortada al ras del suelo.

Tanto el registro de datos del estrato arbóreo y arbustivo como el muestreo del estrato herbáceo y hojarasca se efectuaron en mayo y junio de 2016.

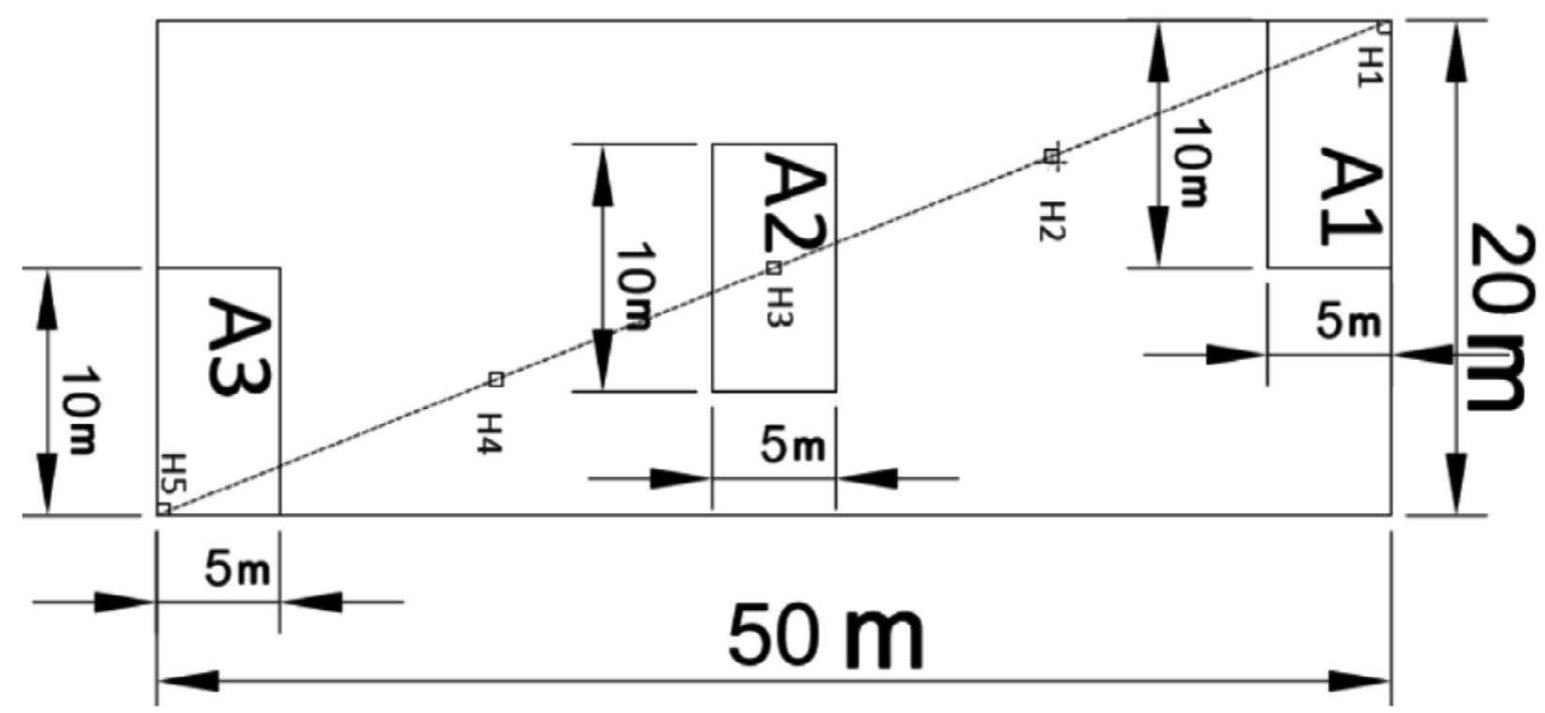

Figura 2. Diseño de la parcela temporal adoptada para la medición y toma de muestra de estrato arbóreo, arbustivo (A1, A2 y A3), herbáceo y hojarasca ( $\mathrm{H} 1, \mathrm{H} 2, \mathrm{H} 3, \mathrm{H} 4$ y H5).

Figure 2. Design of temporal plot laid out for the measurement and sampling of trees, shrubs (A1, A2 and A3) and the herbaceous and litter layers ( $\mathrm{H} 1, \mathrm{H} 2, \mathrm{H} 3, \mathrm{H} 4$ and $\mathrm{H} 5)$.

\subsection{Estimación de carbono}

La biomasa aérea (AGB) de los individuos de árboles y Guadua aff. chacoensis fue estimada mediante la Ecuación 1 (Sato et al., 2015) y Ecuación 2 (Mognon et al., 2014), respectivamente.
$\mathrm{AGB}=0.1739 \times(\mathrm{DAP})^{2.3218}$
$[\mathrm{kg}, \mathrm{cm}]($ E. 1)
$\mathrm{AGB}=-1.70418+0.30633 \times(\mathrm{DAP})^{2}$
$[\mathrm{kg}, \mathrm{cm}]$ (E. 2)

La AGB de las palmeras fue estimada aplicando la Ecuación 3. Para el efecto, se emplearon datos del DAP, Hstem y la proporción de masa seca (dmf) determinada para las especies de palmeras de la Amazonía (0.370) (Goodman et al., 2013).
$\mathrm{AGB}^{0.25}=0.55512 \times\left(\mathrm{dmf} \times \mathrm{DAP}^{2} \times \mathrm{H} \text { stem }\right)^{0.25}$
$[\mathrm{kg}, \mathrm{g} / \mathrm{g}, \mathrm{cm}, \mathrm{m}]$ (E. 3)

La estimación de AGB de los arbustos, excluyendo la mandioca, fue realizada mediante la Ecuación 1. Mientras que la AGB de los individuos de mandioca se determinó mediante la

\begin{tabular}{|c|c|c|}
\hline 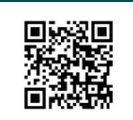 & (c) (i) () () & 24 \\
\hline
\end{tabular}




\section{Revista de CIENCIAS AMBIENTALES Tropical Journal of Environmental Sciences}

Revista de Ciencias Ambientales (Trop J Environ Sci) e-ISSN: 2215-3896

(Julio-Diciembre, 2021) . Vol 55(2): 19-44 DOI: https://doi.org/10.15359/rca.55-2.2 Open Access: www.revistas.una.ac.cr/ambientales e-mail: revista.ambientales@una.ac.cr Kubota V., Pulleman M., Domínguez A., Montiel C., Pineda Y., Ortiz A., Kubsch N., Salas D. y Galeano P.

Ecuación 4, desarrollada para este estudio empleando datos de DAP y altura total $(\mathrm{H})$ de 12 plantas de mandioca provenientes de una plantación ubicada en el Departamento Central de Paraguay $\left(\mathrm{R}^{2}=0.737\right)$. La Ecuación 4 fue aplicada a los 30 individuos de mandioca de cada punto de muestreo y el valor medio fue multiplicado por la cantidad total de plantas registradas en ese punto.

$\mathrm{AGB}=0.3977 \times \mathrm{DAP} \times \mathrm{H}$

$[\mathrm{kg}, \mathrm{cm}, \mathrm{cm}](\mathbf{E} .4)$

Se consideró un almacenamiento de carbono de los árboles y arbustos de $47 \%$ de la AGB (McGroddy et al., 2004). La sumatoria de almacenamiento de carbono de todos los individuos de cada estrato de cada punto de muestreo fue extrapolada a hectárea $(\mathrm{MgC} / \mathrm{ha})$.

Las muestras del estrato herbáceo y hojarasca fueron secadas a $65^{\circ} \mathrm{C}$ hasta alcanzar un peso seco constante. Posteriormente, las impurezas fueron removidas empleando un tamiz de $2 \mathrm{~mm}$. Se obtuvo una muestra compuesta de estrato herbáceo y hojarasca para cada punto de muestreo mediante la mezcla de muestras provenientes de las subparcelas $(\mathrm{H} 1, \mathrm{H} 2, \mathrm{H} 3, \mathrm{H} 4$ y H5) y se determinó el peso seco. Posteriormente, cada muestra compuesta fue triturada y se procedió a la determinación del carbono orgánico por el método Walkley-Black. El contenido de carbono de estos estratos en cada punto de muestreo se determinó mediante el producto del peso seco y el almacenamiento de carbono determinado en el laboratorio. El resultado fue extrapolado a hectárea $(\mathrm{MgC} / \mathrm{ha})$.

\subsection{Muestreo de suelo y análisis en campo}

En cada punto se tomaron muestras compuestas de suelo, para lo cual se aplicó un diseño de parcela con 4 subparcelas (Figura 3). En la subparcela 1 se realizó una calicata de $1 \times 1 \mathrm{~m} \times$ $60 \mathrm{~cm}$. Las subparcelas 2, 3 y 4 fueron ubicadas a una equidistancia de $10 \mathrm{~m}$ desde la subparcela $1\left(0^{\circ}, 120^{\circ}\right.$ y $\left.240^{\circ}\right)$.

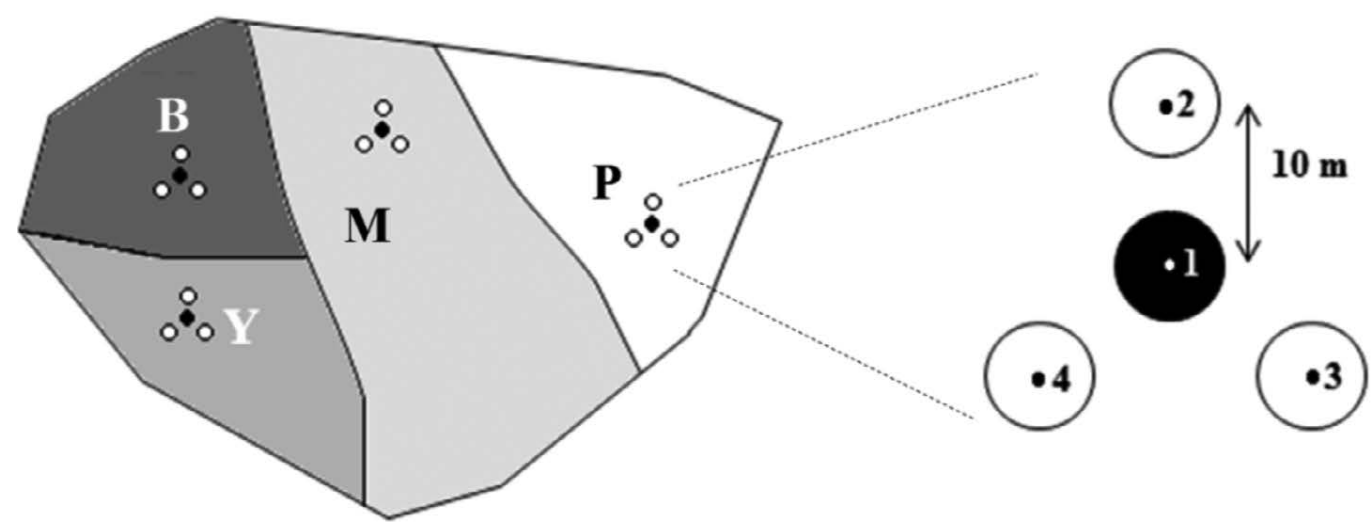

Figura 3. Diseño de muestreo de suelo dentro de una finca con diferentes usos: bosques nativos alterados (B), sistema agroforestal con yerba mate $(\mathrm{Y})$, cultivos de subsistencia $(\mathrm{M})$ y pasturas $(\mathrm{P})$

Figure 3. Soil sampling design within a farm with different land uses: disturbed native forests (B), agroforestry system with yerba mate $(\mathrm{Y})$, subsistence crops $(\mathrm{M})$ and pastures $(\mathrm{P})$.

\begin{tabular}{|c|c|c|c|c|}
\hline 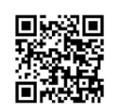 & (c) () & $\underset{\text { AMBEETILIES }}{3}$ & $\frac{1 \%}{2 \%}$ & 25 \\
\hline
\end{tabular}




\section{Revista de CIENCIAS AMBIENTALES Tropical Journal of Environmental Sciences}

Revista de Ciencias Ambientales (Trop J Environ Sci) e-ISSN: 2215-3896

(Julio-Diciembre, 2021) . Vol 55(2): 19-44 DOI: https://doi.org/10.15359/rca.55-2.2

Open Access: www.revistas.una.ac.cr/ambientales e-mail: revista.ambientales@una.ac.cr Kubota V., Pulleman M., Domínguez A., Montiel C., Pineda Y., Ortiz A., Kubsch N., Salas D. y Galeano P.

Para determinar las propiedades químicas y físicas del suelo, fueron tomadas submuestras de tres profundidades de suelo $(0-10,10-20$ y 20-50 cm) en las 4 subparcelas (Figura 3). En cada subparcela se consideró un área de $2.5 \mathrm{~m}$. Se empleó un barreno cilíndrico columnar de $2 \mathrm{~cm}$ de diámetro y se tomaron 4 muestras por profundidad.

Para determinar la densidad aparente $\left(\mathrm{Da}, \mathrm{Mg} / \mathrm{m}^{3}\right)$, se tomaron 2 muestras sin disturbar por profundidad en 4 profundidades $(2.5-7.5,12.5-17.5,25-30$ y $40-45 \mathrm{~cm})$ de una de las paredes de la calicata, usando un cilindro de $5 \mathrm{~cm}$ de diámetro y $5 \mathrm{~cm}$ de altura (Figura 3).

La infiltración fue determinada como indicador de erosión de suelo, mediante el método "time to pond" (Castellanos-Navarrete et al., 2012) en las 4 subparcelas (Figura 3). Para el efecto, se derramó agua a velocidad constante $(0.15 \mathrm{l} / \mathrm{s})$ desde una altitud de $75 \mathrm{~cm}$ en el centro de un anillo de $60 \mathrm{~cm}$ de diámetro y se cronometró el tiempo hasta que el agua rebosara el anillo.

Las muestras de suelo fueron colectadas en mayo y junio de 2016 y los análisis de laboratorio se llevaron a cabo entre septiembre de 2016 y abril 2017.

\subsection{Análisis de suelo en laboratorio}

Las propiedades químicas analizadas fueron: acidez activa $(\mathrm{pH})$, carbono orgánico $(\mathrm{CO}$, $\%)$, nitrógeno total $(\mathrm{N}, \%)$, fósforo disponible $(\mathrm{P}, \mathrm{mg} / \mathrm{kg})$, bases intercambiables [potasio $\left(\mathrm{K}^{+}\right.$, cmolc $/ \mathrm{kg})$, calcio $\left(\mathrm{Ca}^{+2}, \mathrm{cmolc} / \mathrm{kg}\right)$, magnesio $\left(\mathrm{Mg}^{+2}, \mathrm{cmolc} / \mathrm{kg}\right)$, sodio $\left.\left(\mathrm{Na}^{+}, \mathrm{cmolc} / \mathrm{kg}\right)\right]$, acidez potencial $\left(\mathrm{Al}^{+3}+\mathrm{H}^{+}\right.$, cmolc/kg), y capacidad de intercambio catiónico (CIC, cmolc/kg) (National Soil Survey Center, 1996; Tedesco et al., 1995). Además, se calculó la materia orgánica (MO, $\%)$ usando datos de CO. Las propiedades físicas analizadas fueron: el porcentaje de arena, limo y arcilla (\%) por el método de Bouyoucos y la DA por el método del cilindro.

Empleando los resultados de laboratorio de $\mathrm{CO}$ y DA, además de diferentes profundidades del suelo (Ps), fue calculado el carbono orgánico total en suelo por superficie hasta una profundidad de $50 \mathrm{~cm}$ (COS, Ecuación 5). Las DA determinadas a profundidades de 2.5-7.5, 12.5-17.5 y el promedio de 25-30 y 40-45 cm se emplearon para el cálculo correspondiente a la profundidad de $0-10,10-20$ y $20-50 \mathrm{~cm}$, respectivamente.

$$
\mathrm{COS}=\mathrm{CO} \times \mathrm{DA} \times \mathrm{Ps} \quad\left[\mathrm{Mg} / \mathrm{ha}, \%, \mathrm{Mg} / \mathrm{m}^{3}, \mathrm{~m}\right] \text { (E. 5) }
$$

Las muestras compuestas de $0-10 \mathrm{~cm}$ de profundidad también fueron empleadas para determinar la proporción de materia orgánica particulada (MOP, diámetro $\geq 2 \mathrm{~mm}$ ), macroagregados estables (diámetro $\geq 250$ micras) y microagregados (diámetro 53 - 250 micras). Para el efecto se hizo uso de la metodología de fraccionamiento de suelo (Six et al., 2000).

\subsection{Análisis estadístico}

Se determinaron la densidad de árboles, el número de familias botánicas, la a diversidad: riqueza de especies, el índice de diversidad de Simpson $\left(=1-\sum \mathrm{p}_{\mathrm{i}}^{2}\right.$; donde $\mathrm{p}_{\mathrm{i}}$ es abundancia proporcional de la especie i) y el de Shannon - Wiener $\left(\mathrm{H}^{\prime}=-\sum \mathrm{p}_{\mathrm{i}} \log _{2} \mathrm{p}_{\mathrm{i}}\right)$ y la $\gamma$ diversidad: cantidad total de especies en todos los puntos de muestreo. Para evaluar la similitud de la composición

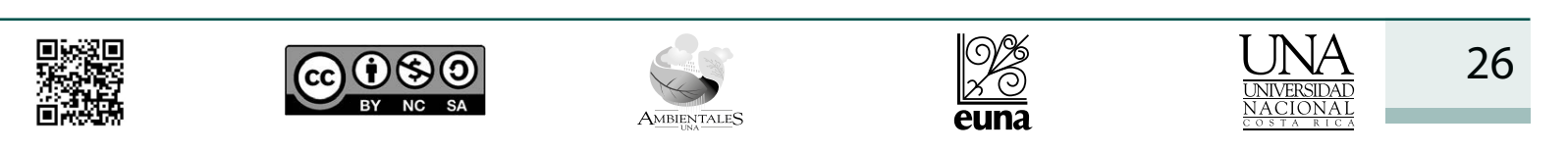




\section{Revista de CIENCIAS AMBIENTALES Tropical Journal of Environmental Sciences}

Revista de Ciencias Ambientales (Trop J Environ Sci) e-ISSN: 2215-3896

(Julio-Diciembre, 2021) . Vol 55(2): 19-44 DOI: https://doi.org/10.15359/rca.55-2.2

Open Access: www.revistas.una.ac.cr/ambientales e-mail: revista.ambientales@una.ac.cr Kubota V., Pulleman M., Domínguez A., Montiel C., Pineda Y., Ortiz A., Kubsch N., Salas D. y Galeano P.

arbórea entre los puntos de muestreo, se calculó el coeficiente de similitud cuantitativo de Bray-Curtis. Subsecuentemente, se desarrolló un análisis cluster empleando la matriz de coeficiente Bray-Curtis entre los puntos de muestreo con individuos arbóreos.

La media del almacenamiento de carbono de cada estrato y la media de la sumatoria de los valores de los estratos fueron comparadas entre los sistemas de uso del suelo mediante el análisis Kruskal-Wallis con un nivel de confianza de $95 \%$, seguido por el análisis de Bonferroni con un nivel de confianza de $95 \%$, en caso de identificarse una diferencia significativa.

El valor medio de las propiedades químicas y físicas del suelo de los 4 sistemas de uso del suelo fue comparado mediante el análisis de Kruskal-Wallis con un nivel de confianza de $95 \%$. En caso de identificarse una diferencia significativa, se aplicó el test de Bonferroni con un nivel de confianza de 95 \%. Para visualizar la proximidad de las propiedades del suelo en función de los sistemas de uso del suelo y los puntos de muestreo, se aplicó el análisis de escalamiento multidimensional no métrico (NMDS), para los datos de propiedades químicas ( $\mathrm{pH}, \mathrm{CO}, \mathrm{N}, \mathrm{P}$, $\mathrm{K}^{+}, \mathrm{Ca}^{+2}, \mathrm{Mg}^{+2}, \mathrm{Na}^{+}, \mathrm{Al}^{+3}+\mathrm{H}^{+}, \mathrm{CIC}$ ) y físicas (porcentaje de arena, limo, arcilla, DA, MOP, macroagregados, microagregados, infiltración) de suelos a 0-10 $\mathrm{cm}$ de profundidad. Todos los análisis tuvieron lugar empleando el programa estadístico R versión 3.5.3. Los datos de los cultivos comerciales fueron presentados en forma separada, a modo de referencia.

\section{Resultados}

\subsection{Efectos de los usos del suelo sobre la diversidad de árboles}

La densidad media de los individuos arbóreos fue más elevada en los bosques nativos alterados y sistemas agroforestales con yerba mate que en los cultivos de subsistencia y pasturas ( $\mathrm{p}<$ 0.05, Apéndice 3). Solo 2 de las fincas con cultivo de subsistencia y pastura presentaron individuos arbóreos. Por otra parte, no se encontraron árboles en ninguno de los puntos de muestreo de cultivos comerciales.

En total fueron registradas 71 especies de árboles, de los cuales 51 se localizaban en los bosques alterados, 47 en sistemas agroforestales con yerba mate, 3 en cultivos de subsistencia y 7 en pasturas ( $\gamma$ diversidad, Apéndice 2 y 3 ). La cantidad media de las especies fue significativamente superior en los bosques alterados y en sistemas agroforestales con yerba mate en comparación a los demás usos ( $\mathrm{p}<0.05$, Apéndice 3). Diez de las especies observadas se encuentran amenazadas o en peligro de extinción a nivel nacional (Ministerio del Ambiente y Desarrollo Sostenible [MADES], 2019). Además, se registraron 7 especies introducidas que se hallaban en los bosques nativos alterados y sistemas agroforestales.

De un total de 24 familias botánicas, aquellas con mayor cantidad de individuos fueron las Fabaceae, Malvaceae, Meliaceae, Rutaceae y Anacardiaceae con 160, 69, 53, 52 y 47 individuos en total respectivamente (Apéndice 2). Las familias con mayor cantidad de especies registradas fueron Fabaceae, Myrtaceae, Lauraceae y Meliaceae, con 14, 5, 6 y 6 especies respectivamente.

\begin{tabular}{|c|c|c|c|c|c|}
\hline 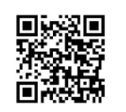 & (c) (i) $(9)$ & $\overbrace{\text { AMBENTILISS }}$ & $\frac{O \%}{2 \%}$ & 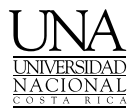 & 27 \\
\hline
\end{tabular}




\section{Revista de CIENCIAS AMBIENTALES Tropical Journal of Environmental Sciences}

Revista de Ciencias Ambientales (Trop J Environ Sci) e-ISSN: 2215-3896

(Julio-Diciembre, 2021) . Vol 55(2): 19-44 DOI: https://doi.org/10.15359/rca.55-2.2 Open Access: www.revistas.una.ac.cr/ambientales e-mail: revista.ambientales@una.ac.cr Kubota V., Pulleman M., Domínguez A., Montiel C., Pineda Y., Ortiz A., Kubsch N., Salas D. y Galeano P.

El valor medio del índice de diversidad de Simpson y de Shannon-Wiener fueron más elevados en los bosques nativos alterados $(0.73 \pm 0.08$ y $2.62 \pm 0.43)$ y sistemas agroforestales con yerba mate $(0.72 \pm 0.06$ y $2.46 \pm 0.46)$ en comparación a los cultivos de subsistencia $(0.25 \pm 0.13$ y $0.50 \pm 0.27)$ y pasturas $(0.61 \pm 0.06$ y $1.52 \pm 0.28)$. Sin embargo, no hubo diferencia estadísticamente significativa ( $\mathrm{p}>0.05$, Apéndice 3).

Se reconocieron 4 grupos de puntos de muestreo mediante el coeficiente de similitud de Bray-Curtis aplicado a datos de composición de especies (Figura 4). El punto B2 estuvo ocupado por Trema micrantha y Guazuma ulmifolia. El segundo grupo (gris claro) tuvo mayor abundancia de Albizia niopoides y Cordia trichotoma. Las especies de mayor abundancia en el tercer grupo (gris oscuro) fueron Peltophorum dubium y Albizia niopoides. El último grupo (negros) estuvo ocupado mayormente por Citrus aurantium, Chrysophyllum marginatum y C. gonocarpum.

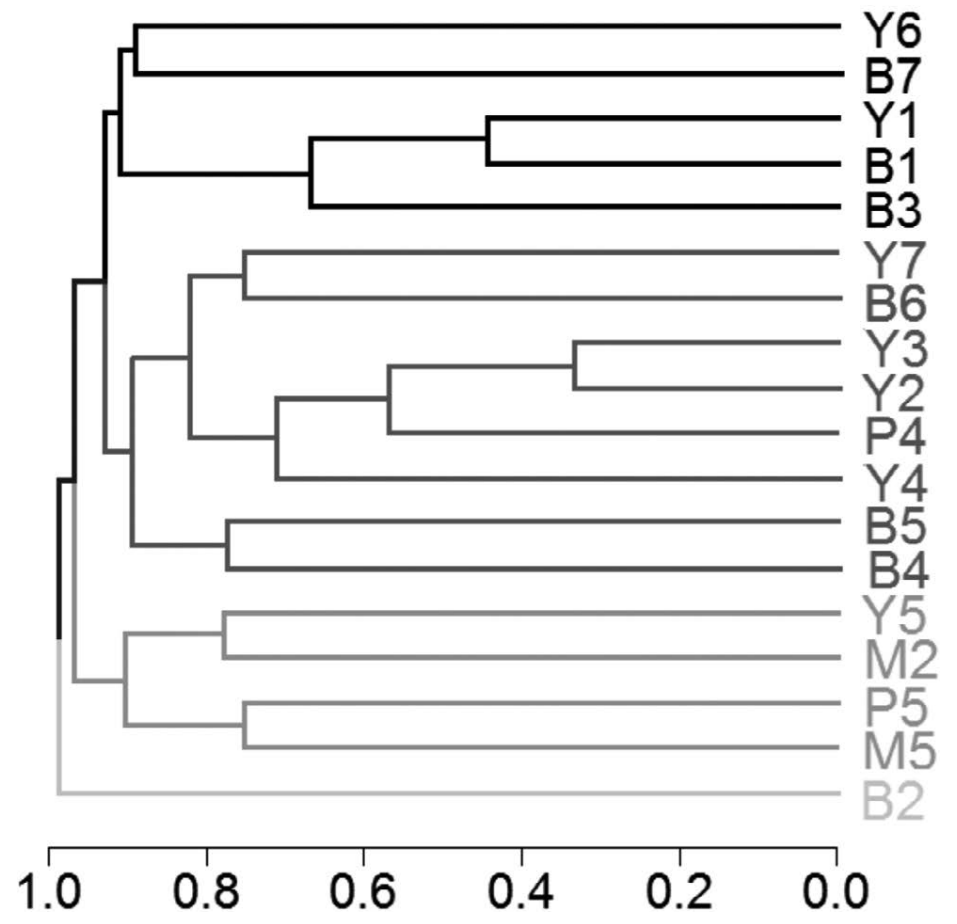

Figura 4. Dendrograma de coeficiente de similitud de Bray-Curtis basado en datos de especies arbóreas de 18 puntos de muestreo: bosques nativos alterados $(\mathrm{B}, \mathrm{n}=7)$, sistema agroforestal con yerba mate $(\mathrm{Y}, \mathrm{n}=7)$, cultivos de subsistencia $(M, n=2)$ y pasturas $(P, n=2)$.

Figure 4. Dendrogram of Bray-Curtis similarity coefficients of species composition from 18 sampling points: disturbed native forests $(B, n=7)$, agroforestry systems with yerba mate $(Y, n=7)$, subsistence crops $(M, n=2)$ and pastures $(\mathrm{P}, \mathrm{n}=2)$.

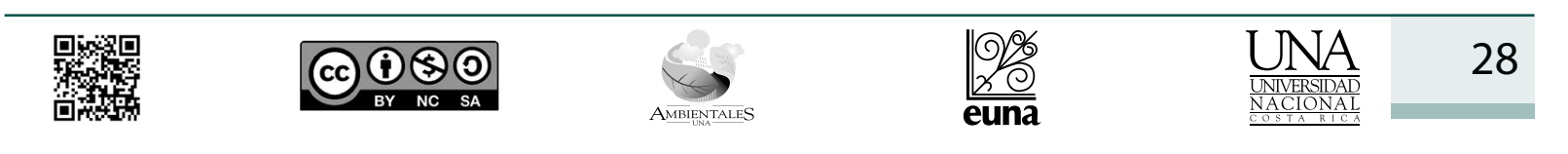




\section{Revista de CIENCIAS AMBIENTALES Tropical Journal of Environmental Sciences}

Revista de Ciencias Ambientales (Trop J Environ Sci) e-ISSN: 2215-3896

(Julio-Diciembre, 2021) . Vol 55(2): 19-44 DOI: https://doi.org/10.15359/rca.55-2.2

Open Access: www.revistas.una.ac.cr/ambientales e-mail: revista.ambientales@una.ac.cr Kubota V., Pulleman M., Domínguez A., Montiel C., Pineda Y., Ortiz A., Kubsch N., Salas D. y Galeano P.

\subsection{Efectos de los usos del suelo en el almacenamiento de carbono aéreo}

Los análisis de laboratorio indicaron que la media del almacenamiento de carbono del estrato herbáceo proveniente de los bosques nativos alterados, sistemas agroforestales con yerba mate, cultivos de subsistencia, pasturas y cultivos comerciales fueron de 49.52, 47.91, 50.12, 50.01 y $43.67 \%$; mientras la media del almacenamiento de carbono de las muestras de hojarasca fueron $42.95,47.78,42.76,51.81$ y $39.5 \%$, respectivamente.

La media del almacenamiento de carbono del estrato arbóreo fue significativamente más alta en los bosques nativos alterados y sistema agroforestal con yerba mate que en otros usos del suelo ( $\mathrm{p}<0.05$, Apéndice 4). La media del contenido de carbono del estrato arbustivo de los bosques nativos alterados fue significativamente más elevada que en otros tipos de uso del suelo $(\mathrm{p}<0.05)$. La media del almacenamiento de carbono del estrato herbáceo de las pasturas fue significativamente más elevada que en otros usos del suelo $(\mathrm{p}<0.05)$.

Los valores más elevados del almacenamiento total de carbono se localizaron en los bosques nativos alterados y sistema agroforestal con yerba mate y no hubo diferencia significativa entre estos dos usos ( $\mathrm{p}<0.05$, Apéndice 4).

Por otra parte, el almacenamiento en las parcelas de cultivos comerciales fue inferior a los sistemas de uso del suelo en las fincas familiares. Fue registrado un total de $1.43 \pm 0.47 \mathrm{MgC} / \mathrm{ha}$, que corresponde a $0.67 \pm 0.22 \mathrm{MgC} /$ ha en el estrato herbáceo y $0.76 \pm 0.27 \mathrm{MgC} / \mathrm{ha}$ en hojarasca.

\subsection{Efectos de los usos del suelo sobre sus propiedades}

En términos de las características intrínsecas del suelo, la textura presentó poca variabilidad tanto entre fincas como dentro de las mismas (Apéndice 5). Todos los puntos muestreados presentaron una clasificación textural arenosa, con un porcentaje de arena próximo a los $80 \%$ en las tres profundidades.

Se observó un aumento de DA a mayor profundidad $\left(1.26,1.34\right.$ y $1.35 \mathrm{Mg} / \mathrm{m}^{3}$ a $0-10,10-20$ y $20-50 \mathrm{~cm}$, respectivamente). A la profundidad de $10-20 \mathrm{~cm}$, la DA de las pasturas fue significativamente superior a la de los bosques nativos alterados ( $\mathrm{p}<0.05$, Apéndice 5). La DA en las pasturas también fue elevada en las demás profundidades, a pesar de que la diferencia no fue significativa ( $\mathrm{p}>0.05)$.

A la profundidad de 0-10 cm, el valor medio de $\mathrm{Ca}^{+2}$ y CIC fue más elevado en los bosques nativos alterados en comparación a las pasturas ( $\mathrm{p}<0.05$, Apéndice 5). La CIC en la pastura fue más bajo en comparación a otros usos. No hubo diferencias significativas entre los diferentes usos del suelo en los demás parámetros químicos ( $\mathrm{p}>0.05$, Apéndice 5). Sin embargo, la pastura presentó valores más bajos en $\mathrm{Mg}^{+2}, \mathrm{~K}^{+}, \mathrm{Na}^{+}$y especialmente $\mathrm{P}$ disponible a la profundidad de 0-10 cm en comparación a otros usos.

No hubo diferencias significativas entre los diferentes usos del suelo en el porcentaje de carbono orgánico y COS ( $\mathrm{p}>0.05$, Apéndice 5). No obstante, a la profundidad de 0-10 cm, el COS fue más elevado en los bosques nativos alterados que en otros usos del suelo (Apéndice 5). Mientras que a la profundidad de 10-20 y 20-50 cm, el COS de las pasturas presentó valores más elevados.

\begin{tabular}{|c|c|c|}
\hline 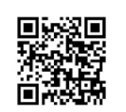 & (c) (i) & 29 \\
\hline
\end{tabular}




\section{Revista de CIENCIAS AMBIENTALES Tropical Journal of Environmental Sciences}

Revista de Ciencias Ambientales (Trop J Environ Sci) e-ISSN: 2215-3896

(Julio-Diciembre, 2021) . Vol 55(2): 19-44 DOI: https://doi.org/10.15359/rca.55-2.2

Open Access: www.revistas.una.ac.cr/ambientales e-mail: revista.ambientales@una.ac.cr Kubota V., Pulleman M., Domínguez A., Montiel C., Pineda Y., Ortiz A., Kubsch N., Salas D. y Galeano P.

El COS $(0-50 \mathrm{~cm})$ de los sistemas agroforestales con yerba mate, cultivo de subsistencia y pasturas representó el 95.3, 94.8 y 114.7 \% del valor observado en los bosques nativos alterados.

No hubo diferencias significativas entre los diferentes usos del suelo en la estabilidad de estructura del suelo ( $\mathrm{p}>0.05$, Apéndice 6). El porcentaje de MOP más bajo fue registrado en los cultivos de subsistencia y el valor más elevado en las pasturas $(\mathrm{p}<0.05)$.

La DA en los cultivos comerciales fue más elevada que en los usos de suelo en la agricultura familiar, siendo sus valores $1.41 \pm 0.04,1.49 \pm 0.02$ y $1.43 \pm 0.02 \mathrm{Mg} / \mathrm{m}^{3}$ a $0-10,10-20$ y $10-50 \mathrm{~cm}$, respectivamente. Sin embargo, los valores de COS fueron bajos $(11.38 \pm 0.97,6.31 \pm 0.66$ y $11.80 \pm$ 1.83). El COS (0-50 cm) en los cultivos comerciales representó el $95.3 \%$ del valor registrado en los bosques nativos alterados. La MOP en los cultivos comerciales fue baja (1.73 \pm 1.27$)$.

A pesar de que las pasturas tuvieron un comportamiento diferente a los demás sistemas de uso, el análisis de NMDS no separó claramente los puntos de muestreo (Figura 5a). Las unidades prediales tuvieron más influencia sobre las propiedades químicas y físicas del suelo que el sistema de uso del suelo (Figura 5b).
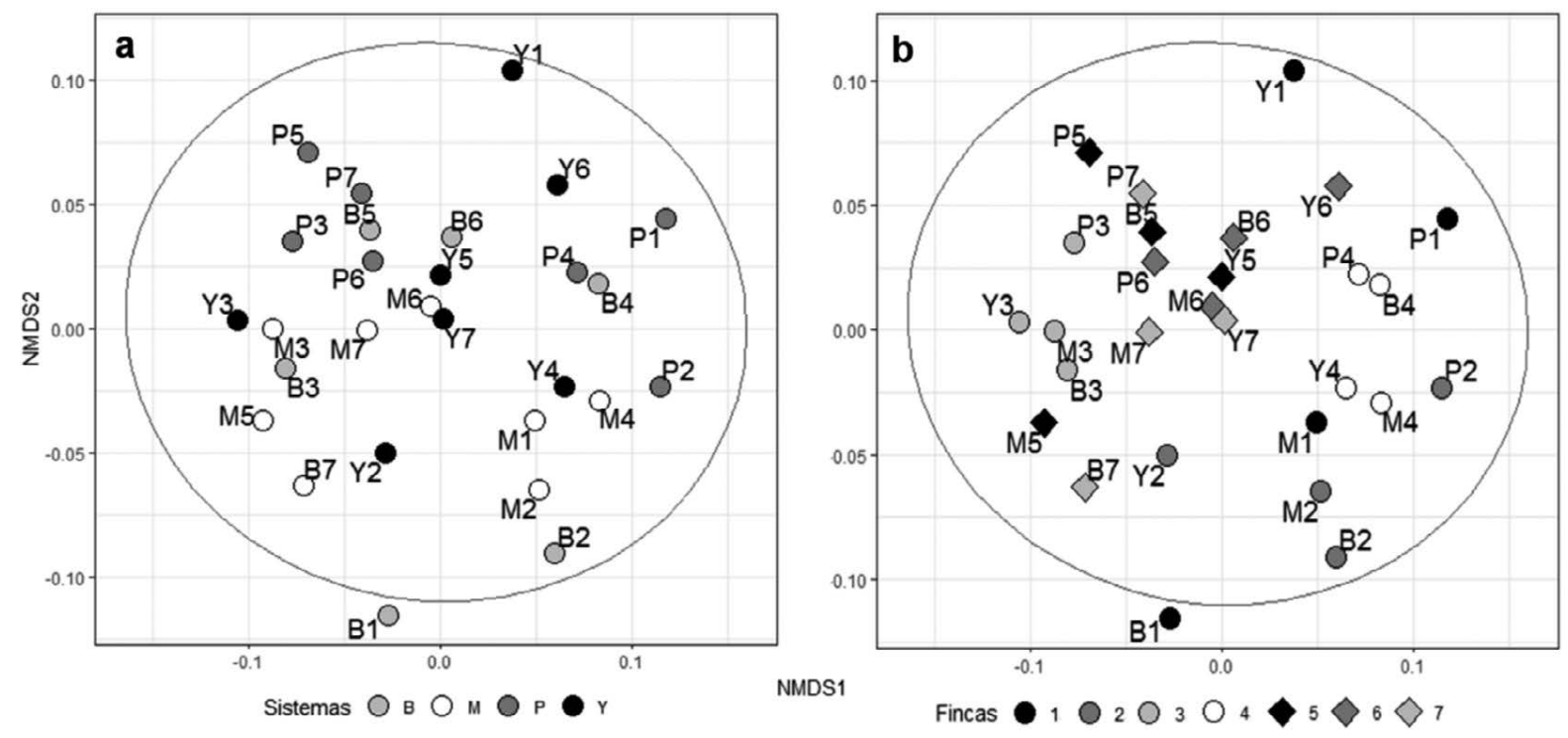

Figura 5. Resultados del análisis de escalamiento multidimensional (NMDS) empleando 10 parámetros químicos y 8 parámetros físicos correspondientes a muestras compuestas de suelo de $0-10 \mathrm{~cm}$ de profundidad de bosques nativos alterados $(\mathrm{B})$, sistema agroforestal con yerba mate $(\mathrm{Y})$, cultivos de subsistencia $(\mathrm{M})$ y pasturas $(\mathrm{P})$, diferenciando a. los sistemas de uso del suelo y b. las fincas. Los elipses indican el intervalo de confianza de $95 \%$. Figure 5. Results of multidimensional scaling (NMDS) using 10 chemical parameters and 8 physical parameters corresponding to composed samples at $0-10 \mathrm{~cm}$ soil depth of disturbed native forests (B), agroforestry system with yerba mate $(\mathrm{Y})$, subsistence crops $(\mathrm{M})$ and pasture $(\mathrm{P})$, indicating $\mathbf{a}$. the land use systems and $\mathbf{b}$. the farms. The ellipses indicate $95 \%$ confidence interval.

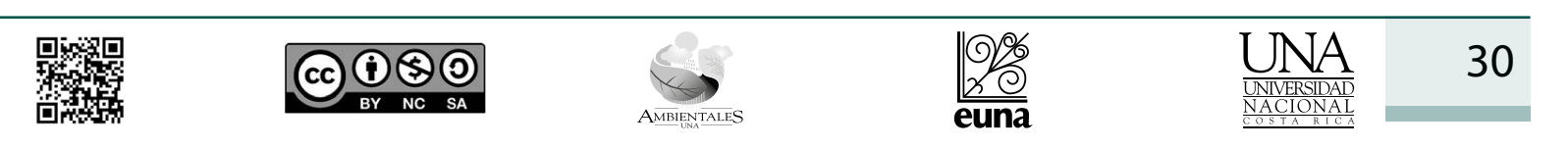




\section{Revista de CIENCIAS AMBIENTALES Tropical Journal of Environmental Sciences}

Revista de Ciencias Ambientales (Trop J Environ Sci) e-ISSN: 2215-3896

(Julio-Diciembre, 2021) . Vol 55(2): 19-44 DOI: https://doi.org/10.15359/rca.55-2.2

Open Access: www.revistas.una.ac.cr/ambientales e-mail: revista.ambientales@una.ac.cr Kubota V., Pulleman M., Domínguez A., Montiel C., Pineda Y., Ortiz A., Kubsch N., Salas D. y Galeano P.

\section{Discusión}

\subsection{Conservación de la diversidad de árboles en la agricultura familiar}

El sistema agroforestal con yerba mate presentó índices de biodiversidad similares a los de los bosques alterados, indicando su potencial para dar un valor económico agregado al bosque y al mismo tiempo contribuir a la conservación de la diversidad arbórea (Apéndice 3). Estos resultados indican que los bosques nativos alterados y los sistemas agroforestales ayudan a conservar las especies forestales dentro del paisaje productivo del BAAPA. Este resultado coincide con las observaciones sobre el aporte de los cultivos agroforestales a la conservación de especies nativas de árboles de la región reportados por De Souza et al. (2012), quienes evaluaron la diversidad de árboles en plantaciones de café (Coffea arabica) bajo bosque en la ecorregión de la Mata Atlántica, reportando una elevada variabilidad en la composición de las especies arbóreas entre sistemas agroforestales de diferentes fincas familiares, lo que contribuye a alcanzar mayor $\gamma$ diversidad que a diversidad dentro del paisaje, al igual que lo observado en este estudio.

Por otra parte, en todos los casos la diversidad de especies arbóreas encontradas en las fincas fue menor en comparación con datos reportados para los bosques nativos de la zona. Álvarez (2016) indicó que el índice de Shannon-Wiener en una parcela permanente de 1 ha instalada en el bosque primario de la Reserva Natural de Bosque Mbaracayú fue de 4.56, mientras que el rango de este índice en los bosques primarios alterados fue de 4.21 a 5.11, y en los bosques secundarios, de 3.67 a 4.76.

La composición de especies arbóreas fue variada entre los puntos de estudio dependiendo del estado de conservación de cada lugar (Apéndice 2). No obstante, en la mayoría de los puntos las especies más abundantes fueron pioneras y heliófitas comunes en bosques explotados, campo abierto y chacras abandonadas, tales como: T. micrantha, G. ulmifolia, A. niopoides, C. trichotoma y P. dubium (López et al., 2002). Solamente en 5 puntos de muestreo (Y6, B7, Y1, B1 y B3) se registró mayor abundancia de especies esciófitas comunes en el estrato medio del bosque en mejor estado de conservación, tales como: C. aurantium, C. marginatum y C. gonocarpum (López et al., 2002).

La reducida biodiversidad registrada en comparación al área silvestre protegida más cercana, la abundancia de especies pioneras y la baja densidad de árboles en algunos puntos de bosque nativo alterado y sistema agroforestal con yerba mate (Ej. B2 y Y5) sugieren la necesidad de estudiar alternativas para enriquecer los remanentes boscosos para maximizar la conservación de las especies nativas de árboles dentro del paisaje productivo. Esta intervención podría llevarse a cabo con especies nativas de importancia económica y de fácil manejo y especies con importancia ecológica y cultural.

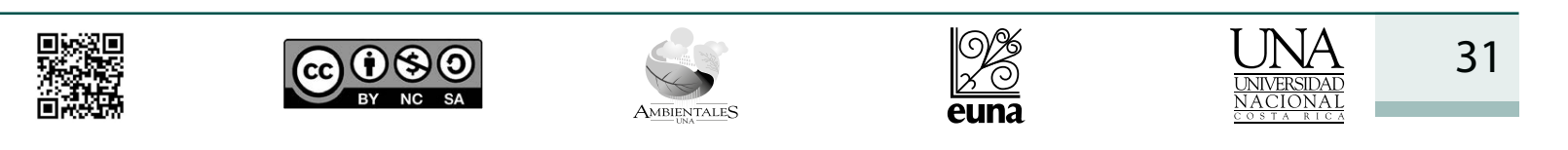




\section{Revista de CIENCIAS AMBIENTALES Tropical Journal of Environmental Sciences}

Revista de Ciencias Ambientales (Trop J Environ Sci) e-ISSN: 2215-3896

(Julio-Diciembre, 2021) . Vol 55(2): 19-44 DOI: https://doi.org/10.15359/rca.55-2.2

Open Access: www.revistas.una.ac.cr/ambientales e-mail: revista.ambientales@una.ac.cr Kubota V., Pulleman M., Domínguez A., Montiel C., Pineda Y., Ortiz A., Kubsch N., Salas D. y Galeano P.

\subsection{Retención de carbono en la agricultura familiar}

El sistema agroforestal con yerba mate presentó un contenido de carbono aéreo similar al de los bosques alterados (Apéndice 4). Estos resultados coinciden con lo planteado por Ramachandran et al. (2010), quienes indicaron que la integración de los árboles en los sistemas de producción agropecuaria permite retener mayor cantidad de carbono por unidad de área, siendo claro su rol en la mitigación del cambio climático. Los valores de almacenamiento de carbono en el estrato arbóreo registrados en los bosques nativos alterados y sistema agroforestal con yerba mate (39.61 y $37.96 \mathrm{MgC} / \mathrm{ha}$ ) cupieron dentro de los rangos indicados para la ecorregión BAAPA, los que para áreas degradadas es de 22.2 - $30.5 \mathrm{MgC} /$ ha y para áreas mejor conservadas de 164.4-189.8 MgC/ha (Facultad de Ciencias Agrarias - Universidad Nacional de Asunción [FCA-UNA], 2013).

El aporte de los bosques nativos alterados y sistemas agroforestales con yerba mate a la retención de carbono aéreo podría mejorar mediante prácticas silviculturales que aceleren el crecimiento de los árboles, como la regulación de densidad de árboles y el enriquecimiento con especies de rápido crecimiento (Ramachandran et al., 2010).

Cabe destacar que el contenido de carbono en los cultivos comerciales fue mucho menor y además podría variar dependiendo de la época y las especies cultivadas. Es de esperar que las fincas con cultivos comerciales presenten mayor almacenamiento de carbono por superficie durante los meses de mayor cobertura vegetal.

Los resultados sugieren que el impacto negativo de los sistemas de producción agropecuaria practicados en las fincas estudiadas sobre el CO del suelo podría ser menor que los reportados para la región, pues se observaron valores elevados de COS $(0-50 \mathrm{~cm})$ en comparación con el carbono aéreo. Además, no se observó diferencia significativa en el CO y COS entre los sistemas del uso del suelo, a pesar de los años de ocupación y uso de las fincas (Apéndice 5), a diferencia del reporte de Riezebos y Loerts (1998), quienes encontraron diferencias significativas entre diferentes usos del suelo del BAAPA. Por otra parte, el aporte relativo de la agricultura de subsistencia y la pastura a la retención de carbono en suelo registrado en este estudio fue más elevado que el valor presentado por Grossman (2015) (Apéndice 5), quien estimó que el contenido de carbono en la plantación de Citrus sp. y yerba mate, la agricultura de subsistencia y la pastura corresponde al $94 \%$, $56 \%$ y $82 \%$ del bosque nativo del BAAPA.

El COS $(0-50 \mathrm{~cm})$ fue relativamente alto en las pasturas debido a una mayor compactación del suelo y contenidos de CO relativamente alto a profundidades mayores a $10 \mathrm{~cm}$.

\subsection{Fertilidad y estructura del suelo en la agricultura familiar}

El alto porcentaje de arena (cerca de $80 \%$ ) no varió entre las fincas y entre usos dentro de la misma finca, sugiriendo que es poco probable que las diferencias en otras propiedades del suelo observadas entre los puntos de muestreo se deban a la variación de granulometría.

La MO es una variable clave para la fertilidad, debido a que es reservorio de nutrientes y afecta positivamente a la CIC. La retención de agua y la estructura del suelo y el carbono

(cc)(i)(2)




\section{Revista de CIENCIAS AMBIENTALES Tropical Journal of Environmental Sciences}

Revista de Ciencias Ambientales (Trop J Environ Sci) e-ISSN: 2215-3896

(Julio-Diciembre, 2021) . Vol 55(2): 19-44 DOI: https://doi.org/10.15359/rca.55-2.2

Open Access: www.revistas.una.ac.cr/ambientales e-mail: revista.ambientales@una.ac.cr Kubota V., Pulleman M., Domínguez A., Montiel C., Pineda Y., Ortiz A., Kubsch N., Salas D. y Galeano P.

representa aproximadamente $58 \%$ de la MO (Heaton et al., 2016). En general los contenidos de $\mathrm{CO}$ en el suelo cambian rápidamente después de la conversión de bosques subtropicales a agricultura (Riezebos y Loerts, 1998). Sin embargo, independiente de los años de uso de los suelos de las fincas (Apéndice 1) no fueron encontradas diferencias significativas en el contenido de materia orgánica de suelos entre los diferentes usos (Apéndice 5). Este resultado puede deberse a la textura arenosa de los suelos que normalmente presentan bajas concentraciones de $\mathrm{MO}$ y nutrientes en general por su baja capacidad de retención de los mismos.

En general, los suelos se caracterizaron por su baja disponibilidad de nutrientes debido a sus características intrínsecas. Se observó que las pasturas presentaron en todos los casos índices de calidad de suelo más bajos y especialmente en comparación con bosques nativos alterados, sugiriendo la relevancia de la cobertura forestal para la mejor conservación de la fertilidad del suelo y capacidad de retención de nutrientes (Apéndice 5). Asimismo, la ausencia de diferencias significativas de calidad del suelo entre los sistemas agroforestales con yerba mate y los sistemas de producción agrícola y pecuaria pudo deberse a la reducida disponibilidad de materia orgánica y hojarasca en comparación al bosque, relacionados con la textura arenosa del suelo. Estos resultados sugieren la necesidad de implementar prácticas de manejo de suelo incluyendo el uso de enmiendas orgánicas.

Las propiedades del suelo a $0-10 \mathrm{~cm}$ de profundidad de los bosques nativos alterados y sistemas agroforestales con yerba mate presentaron tendencias similares a los resultados reportados por Velázquez et al. (2019), quienes en un estudio realizado en la RBBM encontraron que, en promedio, los valores de $\mathrm{pH}, \mathrm{MO}, \mathrm{P}, \mathrm{Ca}^{+2}, \mathrm{Mg}^{+2} \mathrm{y} \mathrm{Na}^{+}$fueron mayores en los bosques remanentes en comparación al sistema agroforestal con yerba mate. Los mismos autores sugirieron que esas diferencias podrían estar relacionadas a la mayor abundancia y diversidad de mesofauna en la hojarasca de los bosques remanentes. Según Baretta et al. (2011), la fauna edáfica cumple funciones relevantes en el ciclo de nutrientes, descomposición de MO y mejoramiento de propiedades físicas de los suelos.

Los resultados sobre la DA, porcentaje de macroagregados mayores e infiltración indican que el suelo de los bosques nativos alterados está en mejores condiciones físicas en comparación a los demás usos del suelo (Apéndices 5 y 6). Los sistemas agroforestales presentaron valores similares a los registrados en los bosques, demostrando que favorece la conservación del suelo.

\subsection{Implicaciones para servicios ecosistémicos en paisajes heterogéneos}

La agricultura sostenible depende de una variedad de servicios de soporte y contribuye al suministro de servicios ecosistémicos. La exploración e implementación de prácticas que facilitarán el uso eficiente del suelo se basa en un trabajo empírico que evalúa directamente los cambios en la provisión de servicios ecosistémicos en paisajes que se encuentran bajo un manejo heterogéneo (Grossman, 2015). En Paraguay existen pocos estudios sobre las variables estructurales y funcionales de sistemas de producciones sostenibles que afectan los servicios

\begin{tabular}{|c|c|c|}
\hline 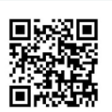 & (c) () () () & 33 \\
\hline
\end{tabular}




\section{Revista de CIENCIAS AMBIENTALES Tropical Journal of Environmental Sciences}

Revista de Ciencias Ambientales (Trop J Environ Sci) e-ISSN: 2215-3896

(Julio-Diciembre, 2021) . Vol 55(2): 19-44 DOI: https://doi.org/10.15359/rca.55-2.2

Open Access: www.revistas.una.ac.cr/ambientales e-mail: revista.ambientales@una.ac.cr Kubota V., Pulleman M., Domínguez A., Montiel C., Pineda Y., Ortiz A., Kubsch N., Salas D. y Galeano P.

del ecosistema y la multifuncionalidad de la agricultura familiar, relevando el presente estudio como aporte importante al conocimiento de los servicios ecosistémicos en la ecorregión.

Los mosaicos de paisajes son cada vez más afectados por la expansión de la agricultura empresarial que ocupa elevada superficie en el paisaje del BAAPA (MAG, 2009). Estos sistemas comerciales no solo demuestran diferencias en términos de escala, sino también en la mecanización (en base de siembra directa) y los tipos de insumos agrícolas empleados. Aunque en este estudio se incluyeron unas referencias de parcelas de cultivos comerciales, debido a limitaciones de diseño no se presentó un análisis robusto de los impactos de estos sistemas sobre los servicios ecosistémicos.

Finalmente, se resalta la importancia de realizar estudios sobre los trade-offs y sinergias entre la conservación, los servicios ecosistémicos y los medios de vida proporcionados por los diferentes agroecosistemas de la ecorregión del BAAPA y sus implicancias (pagos por servicios ecosistémicos y/o la conservación de bosques).

\section{Conclusiones}

Los bosques nativos alterados y los sistemas agroforestales con yerba mate aportan al suministro de los servicios ecosistémicos estudiados. Estos sistemas de uso del suelo presentan mayor diversidad arbórea, retención de carbono aéreo y mejor calidad del suelo. Los sistemas agroforestales podría ser una estrategia de doble objetivo (conservación y la generación de ingresos económicos). El enriquecimiento de bosques nativos alterados y sistemas agroforestales con yerba mate y especies de interés económico, ecológico y cultural podría aumentar los servicios ecosistémicos brindados. El contenido de carbono del suelo fue en general elevado y no se observaron diferencias entre sistemas de uso del suelo, a pesar del avanzado año de ocupación y uso de las fincas.

Futuros trabajos deberían considerar los trade-offs y sinergias entre la conservación, los servicios ecosistémicos y los medios de vida proporcionados por los diferentes agroecosistemas de la ecorregión del BAAPA y avanzar en el desarrollo de políticas públicas que permitan un desarrollo sostenible de la zona en términos económicos, sociales y ambientales.

\section{Agradecimientos}

A los productores de la RBBM, quienes permitieron realizar el estudio y brindaron información. A los empleados de la Fundación Moisés Bertoni, quienes apoyaron los trabajos de campo. A la Facultad de Ciencias Agrarias de la Universidad Nacional de Asunción por su disposición en la toma y procesamiento de muestras. Este estudio fue financiado por el Ministerio Federal para la Cooperación Económica y el Desarrollo de Alemania (BMZ) a través del proyecto concedido al Centro Internacional de Agricultura Tropical (CIAT) bajo número de contrato: 81157480 "Addressing the challenges of smallholder farming communities: Restoring Degraded Agroecosystems". Finalmente, agradecemos a la Revista y las personas revisoras anónimas por los comentarios a la versión final del manuscrito.

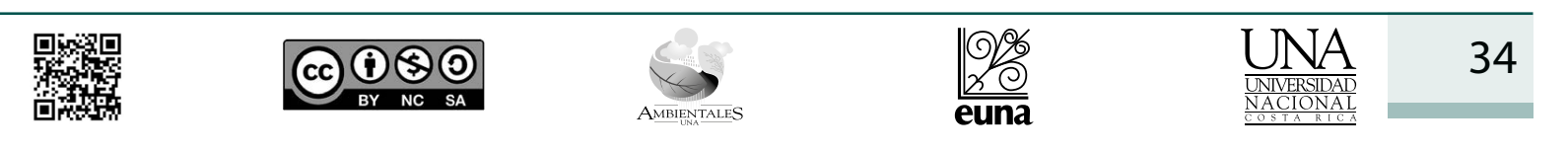




\section{Revista de CIENCIAS AMBIENTALES Tropical Journal of Environmental Sciences}

Revista de Ciencias Ambientales (Trop J Environ Sci) e-ISSN: 2215-3896

(Julio-Diciembre, 2021) . Vol 55(2): 19-44 DOI: https://doi.org/10.15359/rca.55-2.2 Open Access: www.revistas.una.ac.cr/ambientales e-mail: revista.ambientales@una.ac.cr Kubota V., Pulleman M., Domínguez A., Montiel C., Pineda Y., Ortiz A., Kubsch N., Salas D. y Galeano P.

\section{7. Ética y conflicto de intereses}

Las personas autoras declaran que han cumplido totalmente con todos los requisitos éticos y legales pertinentes, tanto durante el estudio como en la producción del manuscrito; que no hay conflictos de intereses de ningún tipo; que todas las fuentes financieras se mencionan completa y claramente en la sección de agradecimientos; y que están totalmente de acuerdo con la versión final editada del artículo.

\section{Referencias}

Álvarez W., G. P. (2016). Análisis comparativo de la composición y estructura de las formaciones boscosas en la Reserva Natural de Bosque Mbaracayú, con énfasis en bosque primario [Tesis de grado no publicada]. Universidad Nacional de Asunción.

Baretta, D., Pires Santos, J. C., Corá Segat, J., Vera Geremia, E., Oliveira Filho, L. C. I., Alves, M. V. (2011). Fauna edáfica e qualidade do solo. En: O. Klauberg Filho, A. L. Mafra (org.), Tópicos em Ciência do Solo (pp. 119-170). Viçosa: Sociedade Brasileira de Ciência do Solo.

Carlson, M. J., Mitchell, R., Rodríguez, L. (2011). Scenario analysis to identify viable conservation strategies in Paraguay's imperiled Atlantic Forest. Ecology and society, 16(3), Artículo 8. http://dx.doi.org/10.5751/ES-04267-160308

Cartes, J. L. (2005). Breve historia de la conservación en el Bosque Atlántico. En. J. L. Cartes (ed.), El bosque atlántico en Paraguay: biodiversidad, amenazas y perspectivas (pp. 37-57). Asociación Guyra Paraguay, Conservation International, Center for Applied Biodiversity Science.

Castellanos-Navarrete, A., Rodriguez-Aragonés, C., Goede, R. G. M. de, Kooistra, M. J., Sayre, K. D., Brussaard, L., Pulleman, M. M. (2012). Earthworm activity and soil structural changes under conservation agriculture in central Mexico. Soil \& Tillage Research, 123, 61-70. https:// doi.org/10.1016/j.still.2012.03.011

De Souza, H. N., Goede, R. G. M., Brussaard, L., Cardoso, I. M., Duarte, E. M. G., Fernandes, R. B. A., Gomes, L. C. y Pulleman, M. M. (2012). Protective shade, tree diversity and soil properties in coffee agroforestry systems in the Atlantic Rainforest biome. Agriculture, Ecosystems and Environment, 146, 179-196. https://doi.org/10.1016/j.agee.2011.11.007.

De Sy, V., Herold, M., Achard, F., Beuchle, R., Clevers, J. G. P. W., Lindquist, E. y Verchot, L. (2015). Land use patterns and related carbon losses following deforestation in South America. Environmental Research Letters, 10, 124004. https://doi.org/10.1088/1748-9326/10/12/124004

Di Bitetti, M., Placci, G. y Dietz, L. (2003). A biodiversity vision for the Upper Paraná Atlantic Forest ecoregion: designing a biodiversity conservation landscape and setting priorities for conservation action. Estados Unidos: World Wildlife Fund.

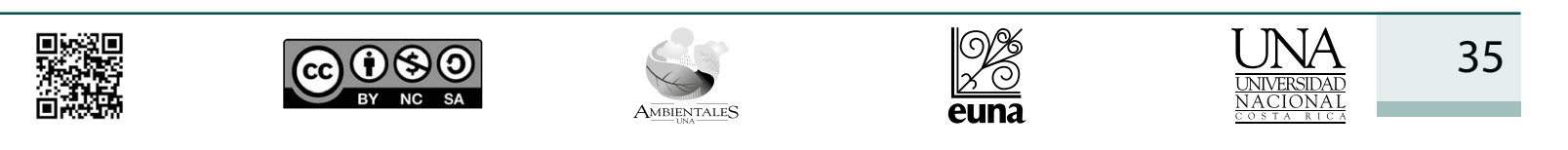




\section{Revista de CIENCIAS AMBIENTALES Tropical Journal of Environmental Sciences}

Revista de Ciencias Ambientales (Trop J Environ Sci) e-ISSN: 2215-3896

(Julio-Diciembre, 2021) . Vol 55(2): 19-44 DOI: https://doi.org/10.15359/rca.55-2.2 Open Access: www.revistas.una.ac.cr/ambientales e-mail: revista.ambientales@una.ac.cr Kubota V., Pulleman M., Domínguez A., Montiel C. Pineda Y., Ortiz A., Kubsch N., Salas D. y Galeano P.

Facultad de Ciencias Agracias-Universidad Nacional de Asunción [FCA-UNA]. (2013). Mapa de stock de carbono bosque atlántico del alto Paraná. https://globallandusechange.org/wp-content/uploads/2017/10/Reporte.-2014.-Carrera-Ingeneria-Forestal-UNA-Desarrollo-del-estudio-de-linea-de-base-para-el-sitio-piloto-Bosque-Atlantico-Alto-Parana.pdf

Fundación Moisés Bertoni para la Conservación de la Naturaleza [FMB]. (2005). Plan de manejo 2005-2010: Reserva Natural del Bosque Mbaracayú. Paraguay.

Galluppi-Selich, T., Villalba, J., Salas-Dueñas, D. A. y Rodríguez, L. (2019). Análisis de la función ecosistémica "protección de acuíferos por cobertura vegetal" en la Reserva de Biósfera del Bosque Mbaracayú (Canindeyú, Paraguay). Revista de la Sociedad Científica del Paraguay, 24(1), 99-113. https://doi.org/10.32480/rscp.2019-24-1.99-113

Goodman, R. C., Phillips, O. L., Del Castillo Torres, D., Freitas, L., Tapia Cortese, S., Monteagudo, A. y Baker, T. R. (2013). Amazon palm biomass and allometry. Forest Ecology and Management, 310, 994-1004. http://dx.doi.org/10.1016/j.foreco.2013.09.045

Grossman, J. J. (2015). Ecosystem service trade-offs and land use among smallholder farmers in eastern Paraguay. Ecology and Society, 20(1), 19. http://dx.doi.org/10.5751/ES-06953-200119

Heaton, L., Fullen, M. A., Bhattacharyya, R. (2016). Critical analysis of the van Bemmelen conversion factor used to convert soil organic matter data to soil organic carbon data: comparative analyses in a UK loamy sand soil. Espaço Aberto, PPGG - UFRJ, 6(1), 35-44. https://doi. org/10.36403/espacoaberto.2016.5244

Huang, C., Kinm, S., Alstatt, A., Townshend, J. R. G., Davis, P., Song, K., Tucker, C. J., Rodas, O., Yanosky, A., Clay, R. y Musinsky, J. (2007). Rapid loss of Paraguay's Atlantic forest and the status of protected area: a Landsat assessment. Remote Sensing of Environment, 106(4), 460466. https://doi.org/10.1016/j.rse.2006.09.016

López, J. A., Little, E. L., Jr., Ritz, J. G. F., Rombold, J. S. y Hahn, W. (2002). Árboles comunes del Paraguay = Nande yvyra mata kuera. Paraguay: Cuerpo de Paz.

López-Gorostiaga, O., González-Erico, E., de Llamas G., P. A., Molinas M., A.S., Franco S., E. S., García S., S. y Ríos A., E. O. (1998). Reconocimiento de suelos y capacidad de uso de la tierra. Región oriental del Paraguay. Paraguay: Ministerio de Agricultura y Ganadería [MAG].

McGroddy, M. E., Daufresne, T. y Hedin, L. O. (2004). Scaling of C:N:P stoichiometry in forests worldwide: Implications of terrestrial Redfield-type ratios. Ecology, 85, 2390-2401. https:// doi.org/10.1890/03-0351

Ministerio de Agricultura y Ganadería [MAG]. (2009). Censo agropecuario nacional 2008: Volumen I. Paraguay. http://www.arp.org.py/images/files/CENSO\%20AGROPECUARIO\%20 2008.pdf

\begin{tabular}{|c|c|}
\hline 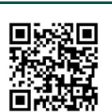 & (c) (i) $\underset{\mathrm{BY}}{\mathrm{NC}} \mathrm{SA}_{\mathrm{SA}}$ \\
\hline
\end{tabular}




\section{Revista de CIENCIAS AMBIENTALES Tropical Journal of Environmental Sciences}

Revista de Ciencias Ambientales (Trop J Environ Sci) e-ISSN: 2215-3896

(Julio-Diciembre, 2021) . Vol 55(2): 19-44 DOI: https://doi.org/10.15359/rca.55-2.2 Open Access: www.revistas.una.ac.cr/ambientales e-mail: revista.ambientales@una.ac.cr Kubota V., Pulleman M., Domínguez A., Montiel C., Pineda Y., Ortiz A., Kubsch N., Salas D. y Galeano P.

Ministerio del Ambiente y Desarrollo Sostenible [MADES]. (2019). Resolución 470/19: Por la que se actualiza el listado de las especies protegidas de la flora silvestre nativa del Paraguay. http:// www.mades.gov.py/resoluciones/por-la-que-se-actualiza-el-listado-de-las-especies-protegidas-de-la-flora-silvestre-nativa-del-paraguay/

Mognon, F., Dalla Corte, A. P., Sanquetta, C. R., Barreto, T. G. y Wojciechowski, J. (2014). Estimativas de biomasa para plantas de bamboo do gênero Guadua. Revista Ceres, 61(6), 900906. http://dx.doi.org/10.1590/0034-737X201461060003

National Soil Survey Center. (1996). Soil Survey Laboratory Methods Manual: Soil Survey Investigations Report $N^{\circ}$ 42. Estados Unidos: United States Department of Agriculture, Natural Resources Conservation Service, National Soil Survey Center.

Ramachandran Nair, P. K., Nair, V. D., Kumar B. M. y Showalter, J. M. (2010). Carbon sequestration in agroforestry system. En: Advances in Agronomy, 108, 237-307. https://doi.org/10.1016/ S0065-2113(10)08005-3

Riezebos, H. y Loerts, A. C. (1998). Influence of land use change and tillage practice on soil organic matter in southern Brazil and eastern Paraguay. Soil and Tillage Research, 49(3), 271 275. https://doi.org/10.1016/S0167-1987(98)00176-7

Sato, T., Saito, M., Ramírez, D., Péres de Molas, L., Toriyam, J., Monda, Y., Kiyono, Y., Herebia, E., Dubie, N., Duré Vera, E., Ramírez Ortega, J. y Vera de Ortiz, M. (2015). Development of Allometric Equations for Tree Biomass in Forest Ecosystems in Paraguay. JARQ, 49(3), 281291. https://doi.org/10.6090/jarq.49.281

Six, J., Elliot, E. T. y Paustian, K. (2000). Soil macroaggregate turnover and microaggregate formation: a mechanism for $\mathrm{C}$ sequestration under no-tillage agriculture. Soil Biology and Biochemistry, 32(14), 2099-20113. https://doi.org/10.1016/S0038-0717(00)00179-6.

Tedesco, M. J., Volkweiss, S. J., Bohnen, H. (1995). Analise de solo, plantas e outros materiais (2da ed.). Brasil: Departamento de solos, UFRGS.

Tortorelli, L. A. (1967). Formaciones forestales y maderas del Paraguay. Boletín del Instituto Forestal Latinoamericano de Investigación y Capacitación, 24, 3-34.

Velázquez Silva, L., C., Kubota, V. R., Ibarra Aranda, J. E. y Salas Dueñas, D. A. (2019). Efectos del cultivo de yerba mate (Ilex paraguariensis) bajo sombra sobre la mesofauna edáfica en la Reserva de Biósfera del Bosque Mbaracayú. Boletín Museo Nacional de Historia Natural del Paraguay, 23(2), 78-89. https://doi.org/10.29327/15304.17-162654

Weinzettel, J., Vačkářů, D., Medková, H. (2018). Human footprint in biodiversity hotspots. Frontiers in Ecology and the Environment, 16(8), 447-452. https://doi.org/10.1002/fee.1825

\begin{tabular}{|c|c|c|}
\hline 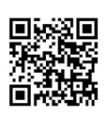 & (c) (1) & 37 \\
\hline
\end{tabular}




\section{Revista de CIENCIAS AMBIENTALES Tropical Journal of Environmental Sciences}

Revista de Ciencias Ambientales (Trop J Environ Sci) e-ISSN: 2215-3896

(Julio-Diciembre, 2021) . Vol 55(2): 19-44 DOI: https://doi.org/10.15359/rca.55-2.2 Open Access: www.revistas.una.ac.cr/ambientales e-mail: revista.ambientales@una.ac.cr Kubota V., Pulleman M., Domínguez A., Montiel C., Pineda Y., Ortiz A., Kubsch N., Salas D. y Galeano P.

Zarza, L. (2017). Paraguay: Situación actual de la agricultura familiar. Políticas públicas y marcos institucionales. En. A. Adib, F. Almada. (coords.) Políticas públicas y marcos institucionales para la agricultura familiar en América Latina (pp. 396-439). Chile: IICA. 


\section{Revista de CIENCIAS AMBIENTALES Tropical Journal of Environmental Sciences}

Revista de Ciencias Ambientales (Trop J Environ Sci)

e-ISSN: 2215-3896

(Julio-Diciembre, 2021) . Vol 55(2): 19-44

DOI: https://doi.org/10.15359/rca.55-2.2

Open Access: www.revistas.una.ac.cr/ambientales

e-mail: revista.ambientales@una.ac.cr

Kubota V., Pulleman M., Domínguez A., Montiel C., Pineda Y., Ortiz A., Kubsch N., Salas D. y Galeano P.

Apéndice 1. Características de las fincas y de los 32 puntos de muestreo en la Reserva de Biosfera del Bosque Mbaracayú. Los datos de superficie total (Sup. total) y años de ocupación (Ocup.) fueron brindados por los propietarios, ocupantes $\mathrm{o}$ arrendatarios de las fincas.

Appendix 1. Characteristics of the farms and 32 selected study sites in Mbaracayú Forest Biosphere Reserve. Data on total surface (Sup. total) and years of occupation (Ocup.) were provided by owners, occupants or tenants of farms.

\begin{tabular}{|c|c|c|c|c|c|c|}
\hline Finca & Comunidad & $\begin{array}{l}\text { Sup. total } \\
\text { de la finca }\end{array}$ & $\begin{array}{l}\text { Ocup. } \\
\text { (años) }\end{array}$ & Código & Uso de suelo & Especies de árboles de mayor abundancia \\
\hline \multirow[t]{4}{*}{1} & \multirow[t]{4}{*}{ Tendal } & \multirow[t]{4}{*}{10 ha } & \multirow[t]{4}{*}{22} & B1 & Bosque nativo alterado & Chrysophyllum marginatum, Citrus aurantium \\
\hline & & & & Y1 & Yerba mate bajo bosque & C. marginatum, C. aurantium \\
\hline & & & & M1 & Cultivo de mandioca & --- \\
\hline & & & & P1 & Pasto Brachiaria aff. brizantha & --- \\
\hline \multirow[t]{4}{*}{2} & \multirow{4}{*}{$\begin{array}{l}11 \mathrm{de} \\
\text { Setiembre }\end{array}$} & \multirow[t]{4}{*}{11 ha } & \multirow[t]{4}{*}{28} & B2 & Bosque nativo alterado & Trema micrantha, Guazuma ulmifolia \\
\hline & & & & $\mathrm{Y} 2$ & Yerba mate en arbustal & Peltophorum dubium, Fabaceae 1 \\
\hline & & & & M2 & Cultivo de mandioca & Albizia niopoides, Cedrela fissilis \\
\hline & & & & $\mathrm{P} 2$ & Pasto Brachiaria aff. brizantha & --- \\
\hline \multirow[t]{4}{*}{3} & \multirow{4}{*}{$\begin{array}{l}11 \mathrm{de} \\
\text { Setiembre }\end{array}$} & \multirow[t]{4}{*}{10 ha } & \multirow[t]{4}{*}{16} & B3 & Bosque nativo alterado & Guarea kunthiana, Lauraceae 3 \\
\hline & & & & Y3 & Yerba mate en arbustal & P. dubium, Parapiptadenia rigida \\
\hline & & & & M3 & Cultivo de mandioca & --- \\
\hline & & & & P3 & Pastura natural & --- \\
\hline \multirow[t]{4}{*}{4} & \multirow[t]{4}{*}{ Tendal } & \multirow[t]{4}{*}{15 ha } & \multirow[t]{4}{*}{33} & B4 & Bosque nativo alterado & G. ulmifolia, Cecropia pachystachya \\
\hline & & & & Y4 & Yerba mate bajo bosque & A. niopoides, Machaerium stipitatum \\
\hline & & & & M4 & Cultivo de mandioca & --- \\
\hline & & & & $\mathrm{P} 4$ & Pastura natural & P. dubium, Albizia niopoides \\
\hline \multirow[t]{4}{*}{5} & \multirow[t]{4}{*}{ Las Residentas } & \multirow[t]{4}{*}{7 ha } & \multirow[t]{4}{*}{23} & B5 & Bosque nativo alterado & Samanea tubulosa, G. ulmifolia \\
\hline & & & & Y5 & Yerba mate bajo bosque & Pterogyne nitens \\
\hline & & & & M5 & Cultivo de mandioca & Cordia trichotoma \\
\hline & & & & P5 & $\begin{array}{l}\text { Mezcla de pastura natural y Brachiaria aff. } \\
\text { brizantha }\end{array}$ & Acrocomia aculeata, C. trichotoma \\
\hline \multirow[t]{4}{*}{6} & \multirow[t]{4}{*}{ Arroyo Guazú } & \multirow[t]{4}{*}{13 ha } & \multirow[t]{4}{*}{18} & B6 & Bosque nativo alterado & Astronium sp., P. dubium \\
\hline & & & & Y6 & Yerba mate bajo bosque & Melia azedarach, Psidium guajava. \\
\hline & & & & M6 & Cultivo de mandioca & --- \\
\hline & & & & P6 & Pastura natural & --- \\
\hline \multirow[t]{4}{*}{7} & \multirow[t]{4}{*}{ Arroyo Guazú } & \multirow[t]{4}{*}{15 ha } & \multirow[t]{4}{*}{18} & B7 & Bosque nativo alterado & Guadua aff. chacoensis, Eucalyptus sp. \\
\hline & & & & Y7 & Plantación de yerba bajo bosque & Balfourodendron riedelianum, $M$. stipitatum, $P$. dubium \\
\hline & & & & M7 & Cultivo de mandioca & --- \\
\hline & & & & P7 & Pasto Brachiaria aff. brizantha & --- \\
\hline 8 & Tendal & 10 ha & 23 & S1 & Cultivo de maíz mecanizado & --- \\
\hline 9 & Tendal & 25 ha & $<20$ & S2 & Cultivo de soja mecanizado & --- \\
\hline 10 & Tendal & $25 \mathrm{ha}^{1}$ & 4 & S3 & Cultivo de soja mecanizado & --- \\
\hline 11 & Arroyo Guazú & & 4 & S4 & Cultivo de soja mecanizado & --- \\
\hline
\end{tabular}

${ }^{1}$ Sumatoria de las propiedades S3 y S4 que corresponden al mismo propietario.

\begin{tabular}{|c|c|c|c|c|}
\hline 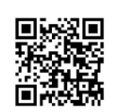 & (c) (1) (5) & $\underset{\text { AMBENETALES }}{\longrightarrow}$ & $\frac{O \%}{20}$ & 39 \\
\hline
\end{tabular}




\section{Revista de CIENCIAS AMBIENTALES Tropical Journal of Environmental Sciences}

Revista de Ciencias Ambientales (Trop J Environ Sci) e-ISSN: 2215-3896

(Julio-Diciembre, 2021) . Vol 55(2): 19-44 DOI: https://doi.org/10.15359/rca.55-2.2

Open Access: www.revistas.una.ac.cr/ambientales e-mail: revista.ambientales@una.ac.cr Kubota V., Pulleman M., Domínguez A., Montiel C., Pineda Y., Ortiz A., Kubsch N., Salas D. y Galeano P.

Apéndice 2. Especies arbóreas registradas en los puntos de muestreo. Appendix 2. Tree species recorded at the sampling points.

\begin{tabular}{|c|c|c|c|c|c|c|}
\hline Familia & Especie & B & $\mathbf{Y}$ & M & $\mathbf{P}$ & Obs. $^{1}$ \\
\hline Anacardiaceae & Astronium aff. urundeuva (Allemão) Engl. var. urundeuva & B6, B7 & Y6 & & & \\
\hline Anacardiaceae & Astronium fraxinifolium Schott var. fraxinifolium & $\mathrm{B} 1$ & $\mathrm{Y} 1, \mathrm{Y} 7$ & & & \\
\hline Anacardiaceae & Mangifera indica $\mathrm{L}$. & B6 & & & & Ex \\
\hline Annonaceae & Annona cacans Warm. & & Y7 & & & $\mathrm{T}$ \\
\hline Annonaceae & Rollinia salicifolia Schltdl. & & Y7 & & & \\
\hline Apocynaceae & Aspidosperma polyneuron Müll. Arg. & $\mathrm{B} 1, \mathrm{~B} 3$ & Y1 & & & $\mathrm{E}$ \\
\hline Apocynaceae & Tabernaemontana catharinensis A. DC. & & $\mathrm{Y} 1, \mathrm{Y} 4$ & & & \\
\hline Arecaceae & Acrocomia aculeata (Jacq.) Lodd. ex Mart. & & $\mathrm{Y7}$ & & P5 & \\
\hline Bignoniaceae & Handroanthus heptaphyllus (Vell.) Mattos & B6 & Y6 & & & $\mathrm{E}$ \\
\hline Bignoniaceae & Jacaranda mimosifolia D. Don. & B3 & & & & \\
\hline Boraginaceae & Cordia americana (L.) Gottschling \& J.S. Mill. & & Y7 & & & \\
\hline Boraginaceae & Cordia ecalyculata Vell. & B7 & & & & \\
\hline Boraginaceae & Cordia trichotoma (Vell.) Arráb. ex Steud. & B5 & $\mathrm{Y} 1, \mathrm{Y} 5, \mathrm{Y} 7$ & M5 & P5 & $\mathrm{E}$ \\
\hline Cannabaceae & Trema micrantha (L.) Blume & B2 & & & & \\
\hline Caricaceae & Carica papaya $\mathrm{L}$ & B7 & & & & Ex \\
\hline Caricaceae & Jacaratia spinosa (Aublet) A. DC. & $\mathrm{B} 1$ & & & & \\
\hline Fabaceae & Albizia niopoides (Spruce ex Benth.) Burkart & $\mathrm{B} 4, \mathrm{~B} 6$ & $\mathrm{Y} 4, \mathrm{Y} 5, \mathrm{Y} 7$ & M2 & $\mathrm{P} 4$ & \\
\hline Fabaceae & Anadenanthera colubrina (Vell.) Brenan var. cebil (Griseb.) Altschul & & Y2 & & $\mathrm{P} 4$ & \\
\hline Fabaceae & Apuleia leiocarpa (Vogel) J.F. Macbr. & B1 & & & & $\mathrm{T}$ \\
\hline Fabaceae & Copaifera langsdorfii var. langsdorfii Desf. & B1 & & & & \\
\hline Fabaceae & Indeterminado 1 & & Y2, Y5, Y7 & & & \\
\hline Fabaceae & Inga aff. uraguensis Hook. \& Arn. & B3, B4 & $\mathrm{Y} 1, \mathrm{Y} 4$ & & & \\
\hline Fabaceae & Machaerium stipitatum (DC) Vogel & B1, B3 & $\mathrm{Y} 1, \mathrm{Y} 4, \mathrm{Y} 7$ & & & \\
\hline Fabaceae & Myrocarpus frondosus Allemão & & & & & $\mathrm{E}$ \\
\hline Fabaceae & Parapiptadenia rigida (Benth.) Brenan & & $\mathrm{Y} 2, \mathrm{Y} 3$ & & & \\
\hline Fabaceae & Peltophorum dubium (Sprengel) Taubert & $\mathrm{B} 3, \mathrm{~B} 5, \mathrm{~B} 6, \mathrm{~B} 7$ & $\mathrm{Y} 1, \mathrm{Y} 2, \mathrm{Y} 3, \mathrm{Y} 4, \mathrm{Y} 6, \mathrm{Y} 7$ & & $\mathrm{P} 4$ & \\
\hline Fabaceae & Pterogyne nitens Tul. & B5, B6, B7 & Y5 & & $\mathrm{P} 4$ & \\
\hline Fabaceae & Samanea tubulosa (Benth.) Barneby \& J.W.Grimes & B4, B5 & & & $\mathrm{P} 4$ & \\
\hline Fabaceae & Senegalia polyphylla (DC.) Britton \& Rose & B7 & & & & \\
\hline Fabaceae & Sweetia fruticosa Sprengel var. fruticosa & B5 & Y4 & & & \\
\hline Lauraceae & Indeterminado 1 & & Y1 & & & \\
\hline Lauraceae & Indeterminado 2 & B5 & & & & \\
\hline Lauraceae & Indeterminado 3 & B3, B5 & Y1 & & & \\
\hline Lauraceae & Indeterminado 4 & & & & & \\
\hline Lauraceae & Nectandra megapotamica (Sprengel) Mez & $\mathrm{B} 1, \mathrm{~B} 3$ & & & & \\
\hline Lauraceae & Ocotea diospyrifolia (Meissner) Mez & $\mathrm{B} 1, \mathrm{~B} 3, \mathrm{~B} 4$ & Y1 & & & \\
\hline Lecythidaceae & Cariniana estrellensis (Raddi) Kuntze & B4 & Y1 & & & $\mathrm{E}$ \\
\hline Loganiaceae & Strychnos brasiliensis (Sprengel) Mart. & & Y7 & & & \\
\hline Malvaceae & Luehea candicans Mart. & $\mathrm{B} 1$ & Y1 & & & \\
\hline Malvaceae & Ceiba speciosa (A.St.-Hil., A.Juss. \& Cambess.) Ravenna & B3 & Y1 & & & \\
\hline Malvaceae & Guazuma ulmifolia Lam. & $\mathrm{B} 2, \mathrm{~B} 4, \mathrm{~B} 5$ & Y4 & & & \\
\hline Meliaceae & Cabralea canjerana (Vell.) Martius & B1, B3 & $\mathrm{Y} 1, \mathrm{Y} 7$ & & & \\
\hline Meliaceae & Cedrela fissilis Vell. & B1, B6 & Y1, Y6, Y7 & M2 & & $\mathrm{E}$ \\
\hline Meliaceae & Guarea kunthiana A. Juss. & B3, B7 & Y1 & & & \\
\hline Meliaceae & Melia azedarach $\mathrm{L}$. & & Y6 & & & Ex \\
\hline
\end{tabular}




\section{Revista de CIENCIAS AMBIENTALES Tropical Journal of Environmental Sciences}

Revista de Ciencias Ambientales (Trop J Environ Sci) e-ISSN: 2215-3896

(Julio-Diciembre, 2021) . Vol 55(2): 19-44 DOI: https://doi.org/10.15359/rca.55-2.2 Open Access: www.revistas.una.ac.cr/ambientales e-mail: revista.ambientales@una.ac.cr Kubota V., Pulleman M., Domínguez A., Montiel C., Pineda Y., Ortiz A., Kubsch N., Salas D. y Galeano P.

\begin{tabular}{|c|c|c|c|c|c|c|}
\hline Familia & Especie & B & $\mathbf{Y}$ & $\mathbf{M}$ & $\mathbf{P}$ & Obs. ${ }^{1}$ \\
\hline Meliaceae & Toona ciliata M. Roem & & Y4 & & & Ex \\
\hline Meliaceae & Trichilia claussenii C. DC. & B3, B7 & & & & \\
\hline Moraceae & Ficus enormis (Mart. ex Miq.) Miq. & B7 & & & & \\
\hline Moraceae & Maclura tinctoria (L.) Steud. ssp. tinctoria & B1 & Y1 & & & \\
\hline Myrtaceae & Campomanesia xanthocarpa O. Berg & & Y7 & & & \\
\hline Myrtaceae & Eucalyptus sp. & B7 & & & & Ex \\
\hline Myrtaceae & Eugenia uniflora L. & B1 & Y1 & & & \\
\hline Myrtaceae & Indeterminado 1 & & & & & \\
\hline Myrtaceae & Indeterminado 2 & & Y1 & & & \\
\hline Myrtaceae & Plinia rivularis (Cambess.) Rotman & B1 & & & & \\
\hline Myrtaceae & Plinia trunciflora (O. Berg.) Kausel & & Y1 & & & \\
\hline Myrtaceae & Psidium guajava $\mathrm{L}$. & & Y6 & & & \\
\hline Poaceae & Guadua aff. chacoensis (Rojas Acosta) Londoño \& P.M. Peterson & B7 & & & & \\
\hline Primulaceae & Myrsine sp. & B3 & & & & \\
\hline Rubiaceae & Genipa americana $\mathrm{L}$. & B1 & & & & \\
\hline Rutaceae & Balfourodendron riedelianum (Engl.) Engl. & B3, B6 & $\mathrm{Y} 4, \mathrm{Y} 7$ & & & $\mathrm{E}$ \\
\hline Rutaceae & Citrus aurantium $\mathrm{L}$. & B3 & Y1, Y6, Y7 & & & Ex \\
\hline Rutaceae & Citrus sinensis (L.) Osbeck, & & Y7 & & & Ex \\
\hline Rutaceae & Helietta apiculata Benth. & B1, B6 & & & & \\
\hline Rutaceae & Zanthoxylum fagara (L.) Sarg. & B1 & Y1 & & & \\
\hline Salicaceae & Casearia sp. & B6, B7 & Y1, Y7 & & & \\
\hline Sapindaceae & Cupania vernalis Cambess. & B1 & Y1 & & & \\
\hline Sapindaceae & Diatenopteryx sorbifolia Radlk. & B1 & & & & \\
\hline Sapotaceae & Chrysophyllum gonocarpum (Martius \& Eichler) Engler & B1, B3 & $\mathrm{Y} 1, \mathrm{Y} 7$ & & & \\
\hline Sapotaceae & Chrysophyllum marginatum (Hooker \& Arnott) Radlkofer subsp. marginatum & $\mathrm{B} 1, \mathrm{~B} 3$ & Y1 & & & \\
\hline Urticaceae & Cecropia pachystachya Trécul & B3, B4, B5 & $\mathrm{Y} 1, \mathrm{Y} 7$ & & & \\
\hline
\end{tabular}

${ }^{1}$ E: especie en peligro (MADES, 2019); AM: especie amenazada (MADES, 2019) y Ex: especie exótica.

Apéndice 3. Densidad de árboles, número de familias y especies arbóreas, porcentaje de cantidad total de especies arbóreas identificadas para todas las fincas, índice de diversidad de Simpson y de Shannon-Wiener, con sus pro medios y errores estándares de los puntos de muestreo.

Appendix 3. Tree density, number of tree families and tree species, percentage of the total number of identified tree species for all farms, Simpson and Shannon-Wiener diversity index, and standard errors at sampling points.

\begin{tabular}{|c|c|c|c|c|c|c|}
\hline \multirow{2}{*}{ Código } & \multirow{2}{*}{$\begin{array}{l}\text { Densidad } \\
\text { (ind./ha) }\end{array}$} & \multirow{2}{*}{$\begin{array}{l}\text { Cantidad de } \\
\text { familias }\end{array}$} & \multirow{2}{*}{$\begin{array}{l}\text { Cantidad de } \\
\text { especies }\end{array}$} & \multirow{2}{*}{$\begin{array}{c}\text { \% de cantidad total } \\
\text { de especies arbóreas } \\
\text { identificadas }\end{array}$} & \multicolumn{2}{|c|}{ Índice de diversidad } \\
\hline & & & & & Simpson & Shannon \\
\hline B1 & 550 & 13 & 23 & 32.39 & 0.93 & 4.14 \\
\hline B2 & 90 & 2 & 2 & 2.82 & 0.44 & 0.92 \\
\hline B3 & 470 & 10 & 18 & 25.35 & 0.84 & 3.36 \\
\hline B4 & 670 & 5 & 7 & 9.86 & 0.47 & 1.41 \\
\hline B5 & 480 & 5 & 9 & 12.68 & 0.81 & 2.61 \\
\hline B6 & 780 & 6 & 10 & 14.08 & 0.74 & 2.54 \\
\hline \multirow[t]{2}{*}{ B7 } & 250 & 9 & 12 & 16.90 & 0.89 & 3.35 \\
\hline & $470 \pm 89.36 \mathbf{a}$ & $7.14 \pm 1.41 \mathrm{a}$ & $11.57 \pm 2.65 \mathbf{a}$ & $16.9 \pm 3.45 \mathrm{a}$ & $0.73 \pm 0.08$ & $2.62 \pm 0.43$ \\
\hline Y1 & 490 & 15 & 28 & 39.44 & 0.94 & 4.43 \\
\hline Y2 & 160 & 1 & 4 & 5.63 & 0.60 & 1.59 \\
\hline 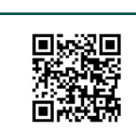 & ( & & $\underset{A M B I E N T A L E S}{2}$ & $\frac{1 \% \text { \% }}{\text { euna }}$ & $\frac{\text { UI }}{\frac{\text { UNIVI }}{\mathrm{NAC}}}$ & 41 \\
\hline
\end{tabular}




\section{Revista de CIENCIAS AMBIENTALES Tropical Journal of Environmental Sciences}

Revista de Ciencias Ambientales (Trop J Environ Sci) e-ISSN: 2215-3896

(Julio-Diciembre, 2021) . Vol 55(2): 19-44 DOI: https://doi.org/10.15359/rca.55-2.2 Open Access: www.revistas.una.ac.cr/ambientales e-mail: revista.ambientales@una.ac.cr Kubota V., Pulleman M., Domínguez A., Montiel C., Pineda Y., Ortiz A., Kubsch N., Salas D. y Galeano P.

\begin{tabular}{|c|c|c|c|c|c|c|}
\hline \multirow[b]{2}{*}{ Código } & \multirow{2}{*}{$\begin{array}{l}\text { Densidad } \\
\text { (ind./ha) }\end{array}$} & \multirow{2}{*}{$\begin{array}{l}\text { Cantidad de } \\
\text { familias }\end{array}$} & \multirow{2}{*}{$\begin{array}{l}\text { Cantidad de } \\
\text { especies }\end{array}$} & \multirow{2}{*}{$\begin{array}{c}\text { \% de cantidad total } \\
\text { de especies arbóreas } \\
\text { identificadas }\end{array}$} & \multicolumn{2}{|c|}{ Î́ndice de diversidad } \\
\hline & & & & & Simpson & Shannon \\
\hline Y3 & 110 & 1 & 2 & 2.82 & 0.46 & 0.95 \\
\hline Y4 & 380 & 5 & 9 & 12.68 & 0.78 & 2.54 \\
\hline Y5 & 70 & 2 & 4 & 5.63 & 0.61 & 1.66 \\
\hline Y6 & 280 & 6 & 7 & 9.86 & 0.78 & 2.43 \\
\hline \multirow[t]{2}{*}{ Y7 } & 680 & 12 & 21 & 29.58 & 0.86 & 3.60 \\
\hline & $310 \pm 83.84 \mathrm{a}$ & $6.00 \pm 2.09 \mathrm{a}$ & $10.71 \pm 3.74 \mathbf{a}$ & $15.09 \pm 4.88 \mathrm{a}$ & $0.72 \pm 0.06$ & $2.46 \pm 0.46$ \\
\hline M1 & 0 & 0 & 0 & 0.00 & - & - \\
\hline M2 & 20 & 2 & 2 & 2.82 & 0.50 & 1.00 \\
\hline M3 & 0 & 0 & 0 & 0.00 & - & - \\
\hline M4 & 0 & 0 & 0 & 0.00 & - & - \\
\hline M5 & 10 & 1 & 1 & 1.41 & 0.00 & 0.00 \\
\hline M6 & 0 & 0 & 0 & 0.00 & - & - \\
\hline \multirow[t]{2}{*}{ M7 } & 0 & 0 & 0 & 0.00 & - & - \\
\hline & $4.29 \pm 2.97 \mathbf{b}$ & $0.43 \pm 0.30 \mathbf{b}$ & $0.43 \pm 0.30 \mathbf{b}$ & $0.6 \pm 0.39 \mathbf{b}$ & $0.25 \pm 0.13$ & $0.5 \pm 0.27$ \\
\hline P1 & 0 & 0 & 0 & 0.00 & - & - \\
\hline $\mathrm{P} 2$ & 0 & 0 & 0 & 0.00 & - & - \\
\hline P3 & 0 & 0 & 0 & 0.00 & - & - \\
\hline $\mathrm{P} 4$ & 120 & 1 & 5 & 7.04 & 0.72 & 2.05 \\
\hline P5 & 70 & 2 & 2 & 2.82 & 0.49 & 0.99 \\
\hline P6 & 0 & 0 & 0 & 0.00 & - & - \\
\hline \multirow[t]{2}{*}{ P7 } & 0 & 0 & 0 & 0.00 & - & - \\
\hline & $27.14 \pm 18.35 \mathbf{b}$ & $0.43 \pm 0.30 \mathbf{b}$ & $1.00 \pm 0.72 \mathbf{b}$ & $1.41 \pm 0.94 \mathbf{b}$ & $0.61 \pm 0.06$ & $1.52 \pm 0.28$ \\
\hline Valor $\mathrm{p}$ & 0.00 & 0.00 & 0.00 & 0.00 & 0.29 & 0.20 \\
\hline
\end{tabular}

Apéndice 4. Comparación de la media y error estándar de la cantidad de carbono ( $\mathrm{MgC} / \mathrm{ha})$ en el estrato arbóreo, arbustivo, herbáceo y hojarasca en bosques nativos alterados (B), sistema agroforestal con yerba mate (Y), cultivos de subsistencia (M) y pasturas (P).

Appendix 4. Comparison of mean of carbon stocks and standard error $(\mathrm{MgC} / \mathrm{ha})$ in tree, shrub, herbaceous and litter layer of disturbed native forests $(\mathrm{B})$, agroforestry systems with yerba mate $(\mathrm{Y})$, subsistence crops $(\mathrm{M})$ and pastures $(\mathrm{P})$.

\begin{tabular}{lrcccccccccccc}
\hline \multicolumn{1}{c}{ Estrato } & & $\mathbf{B}$ & & & $\mathbf{Y}$ & & & $\mathbf{M}$ & & & P & Valor $\mathbf{p}$ \\
\hline Arbóreo & 39.61 & \pm 8.67 & $\mathbf{a}$ & 37.96 & \pm 12.26 & $\mathbf{a}$ & 1.00 & \pm 0.90 & $\mathbf{b}$ & 2.85 & \pm 2.04 & $\mathbf{b}$ & 0.00 \\
Arbustivo & 7.34 & \pm 2.08 & $\mathbf{a}$ & 2.58 & \pm 0.59 & $\mathbf{b}$ & 0.74 & \pm 0.19 & $\mathbf{b}$ & 0.18 & \pm 0.13 & $\mathbf{b}$ & 0.00 \\
Herbáceo & 0.23 & \pm 0.09 & $\mathbf{a}$ & 0.52 & \pm 0.15 & $\mathbf{a}$ & 0.42 & \pm 0.10 & $\mathbf{a}$ & 1.69 & \pm 0.37 & $\mathbf{b}$ & 0.00 \\
Hojarasca & 2.64 & \pm 0.45 & & 2.05 & \pm 0.40 & & 0.92 & \pm 0.17 & & 1.83 & \pm 0.65 & 0.02 \\
Total & 49.81 & \pm 7.73 & $\mathbf{a}$ & 43.11 & \pm 12.45 & $\mathbf{a}$ & 3.06 & \pm 1.03 & $\mathbf{b}$ & 6.54 & \pm 1.89 & $\mathbf{b}$ & 0.00 \\
\hline
\end{tabular}

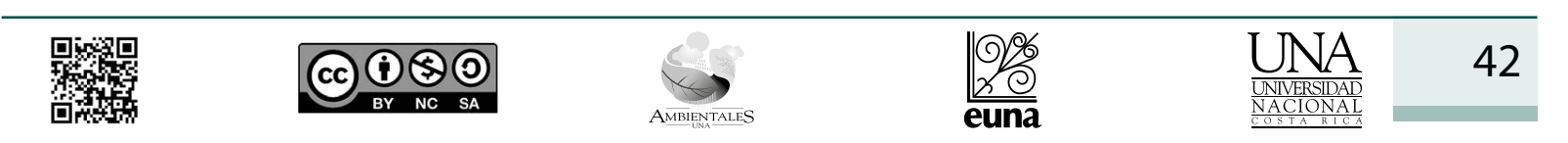




\section{Revista de CIENCIAS AMBIENTALES Tropical Journal of Environmental Sciences}

Revista de Ciencias Ambientales (Trop J Environ Sci) e-ISSN: 2215-3896

(Julio-Diciembre, 2021) . Vol 55(2): 19-44 DOI: https://doi.org/10.15359/rca.55-2.2 Open Access: www.revistas.una.ac.cr/ambientales e-mail: revista.ambientales@una.ac.cr Kubota V., Pulleman M., Domínguez A., Montiel C., Pineda Y., Ortiz A., Kubsch N., Salas D. y Galeano P.

Apéndice 5. Comparación de medias y error estándar de las propiedades químicas y físicas del suelo tomadas a la profundidad de $0-10 \mathrm{~cm}, 10-20 \mathrm{~cm}$ y $20-50 \mathrm{~cm}$ en bosques nativos alterados (B), sistema agroforestal con yerba mate $(\mathrm{Y})$, cultivos de subsistencia $(\mathrm{M})$ y pasturas $(\mathrm{P})$.

Appendix 5. Comparison of means and standard error of chemical and physical properties of soil from $0-10 \mathrm{~cm}$, $10-20 \mathrm{~cm}$ and $20-50 \mathrm{~cm}$ of disturbed native forests (B), agroforestry systems with yerba mate (Y), subsistence crops $(\mathrm{M})$ and pastures $(\mathrm{P})$.

\begin{tabular}{|c|c|c|c|c|c|c|c|c|c|}
\hline $0-10 \mathrm{~cm}$ & \multicolumn{2}{|c|}{ B } & \multicolumn{2}{|c|}{$\mathbf{Y}$} & \multicolumn{2}{|c|}{$\mathbf{M}$} & \multicolumn{2}{|c|}{$\mathbf{P}$} & Valor $\mathbf{p}$ \\
\hline $\mathrm{pH}$ & 5.84 & \pm 0.25 & 4.53 & \pm 0.20 & 5.64 & \pm 0.38 & 5.50 & \pm 0.33 & 0.89 \\
\hline $\mathrm{CO}(\%)$ & 1.07 & \pm 0.14 & 0.94 & \pm 0.13 & 0.86 & \pm 0.11 & 0.92 & \pm 0.10 & 0.55 \\
\hline $\mathrm{MO}(\%)$ & 1.84 & \pm 0.25 & 1.62 & \pm 0.23 & 1.49 & \pm 0.18 & 1.59 & \pm 0.17 & 0.53 \\
\hline $\operatorname{COS}(\mathrm{Mg} / \mathrm{ha})$ & 12.59 & \pm 1.28 & 11.49 & \pm 1.70 & 10.17 & \pm 1.16 & 12.32 & \pm 1.51 & 0.51 \\
\hline $\mathrm{P}(\mathrm{mg} / \mathrm{kg})$ & 4.53 & \pm 1.72 & 2.98 & \pm 0.54 & 3.73 & \pm 1.35 & 1.25 & \pm 0.41 & 0.06 \\
\hline $\mathrm{Ca}^{+2}(\mathrm{cmolc} / \mathrm{kg})$ & 3.18 & $\pm 0.46 \mathrm{a}$ & 2.01 & $\pm 0.23 \mathrm{ab}$ & 2.40 & $\pm 0.25 \mathbf{a b}$ & 1.59 & $\pm 0.21 \mathbf{b}$ & 0.02 \\
\hline $\mathrm{Mg}^{+2}(\mathrm{cmolc} / \mathrm{kg})$ & 0.94 & \pm 0.09 & 0.68 & \pm 0.06 & 0.77 & \pm 0.09 & 0.60 & \pm 0.09 & 0.11 \\
\hline $\mathrm{K}^{+}(\mathrm{cmolc} / \mathrm{kg})$ & 0.22 & \pm 0.09 & 0.18 & \pm 0.17 & 0.17 & \pm 0.05 & 0.17 & \pm 0.02 & 0.98 \\
\hline $\mathrm{Na}^{+}(\mathrm{cmolc} / \mathrm{kg})$ & 0.05 & \pm 0.01 & 0.05 & \pm 0.01 & 0.04 & \pm 0.01 & 0.04 & \pm 0.01 & 0.68 \\
\hline $\mathrm{Al}^{+3}+\mathrm{H}^{+}(\mathrm{cmolc} / \mathrm{kg})$ & 0.31 & \pm 0.18 & 0.54 & \pm 0.20 & 0.54 & \pm 0.28 & 0.58 & \pm 0.28 & 0.85 \\
\hline $\mathrm{N}(\%)$ & 0.05 & \pm 0.00 & 0.05 & \pm 0.01 & 0.04 & \pm 0.00 & 0.05 & \pm 0.00 & 0.80 \\
\hline CIC (cmolc/kg) & 5.10 & $\pm 0.40 \mathrm{a}$ & 3.35 & $\pm 0.39 \mathrm{ab}$ & 2.64 & $\pm 0.34 \mathbf{a b}$ & 2.37 & $\pm 0.34 \mathbf{b}$ & 0.02 \\
\hline $\mathrm{DA}\left(\mathrm{Mg} / \mathrm{m}^{3}\right)$ & 1.21 & \pm 0.07 & 1.22 & \pm 0.04 & 1.18 & \pm 0.03 & 1.34 & \pm 0.06 & 0.22 \\
\hline Arena (\%) & 82.57 & \pm 1.07 & 83.14 & \pm 0.46 & 81.86 & \pm 2.54 & 82.29 & \pm 2.31 & 0.61 \\
\hline Limo (\%) & 6.43 & \pm 0.78 & 6.14 & \pm 0.77 & 6.29 & \pm 0.84 & 6.86 & \pm 1.26 & 0.99 \\
\hline Arcilla (\%) & 11.00 & \pm 0.95 & 10.71 & \pm 0.87 & 11.86 & \pm 1.86 & 10.86 & \pm 1.22 & 0.90 \\
\hline \multicolumn{10}{|l|}{$10-20 \mathrm{~cm}$} \\
\hline $\mathrm{pH}$ & 5.60 & \pm 0.43 & 5.01 & \pm 0.28 & 6.04 & \pm 0.36 & 5.97 & \pm 0.26 & 0.09 \\
\hline $\mathrm{CO}(\%)$ & 0.61 & \pm 0.08 & 0.54 & \pm 0.06 & 0.46 & \pm 0.09 & 0.61 & \pm 0.09 & 0.44 \\
\hline MO (\%) & 1.06 & \pm 0.13 & 0.93 & \pm 0.11 & 0.79 & \pm 0.15 & 1.06 & \pm 0.15 & 0.44 \\
\hline $\operatorname{COS}(\mathrm{Mg} / \mathrm{ha})$ & 7.62 & \pm 0.92 & 6.96 & \pm 0.70 & 6.19 & \pm 1.25 & 8.53 & \pm 1.29 & 0.37 \\
\hline $\mathrm{P}(\mathrm{mg} / \mathrm{kg})$ & 2.47 & \pm 0.52 & 1.89 & \pm 0.53 & 2.69 & \pm 1.03 & 1.42 & \pm 0.37 & 0.59 \\
\hline $\mathrm{Ca}^{+2}(\mathrm{cmolc} / \mathrm{kg})$ & 1.65 & \pm 0.23 & 1.37 & \pm 0.16 & 1.70 & \pm 0.15 & 1.45 & \pm 0.11 & 0.41 \\
\hline $\mathrm{Mg}^{+2}(\mathrm{cmolc} / \mathrm{kg})$ & 0.64 & \pm 0.11 & 0.55 & \pm 0.08 & 0.55 & \pm 0.06 & 0.45 & \pm 0.08 & 0.52 \\
\hline $\mathrm{K}^{+}(\mathrm{cmolc} / \mathrm{kg})$ & 0.17 & \pm 0.07 & 0.18 & \pm 0.09 & 0.11 & \pm 0.02 & 0.14 & \pm 0.04 & 0.99 \\
\hline $\mathrm{Na}^{+}(\mathrm{cmolc} / \mathrm{kg})$ & 0.05 & \pm 0.01 & 0.04 & \pm 0.01 & 0.04 & \pm 0.00 & 0.05 & \pm 0.00 & 0.71 \\
\hline $\mathrm{Al}^{+3}+\mathrm{H}^{+}(\mathrm{cmolc} / \mathrm{kg})$ & 0.62 & \pm 0.26 & 0.85 & \pm 0.34 & 0.22 & \pm 0.18 & 0.13 & \pm 0.14 & 0.17 \\
\hline $\mathrm{N}(\%)$ & 0.03 & \pm 0.00 & 0.02 & \pm 0.00 & 0.02 & \pm 0.00 & 0.03 & \pm 0.01 & 0.59 \\
\hline CIC (cmolc/kg) & 2.64 & \pm 0.34 & 2.25 & \pm 0.20 & 2.56 & \pm 0.17 & 2.17 & \pm 0.22 & 0.43 \\
\hline $\mathrm{DA}\left(\mathrm{Mg} / \mathrm{m}^{3}\right)$ & 1.25 & $\pm 0.03 \mathrm{a}$ & 1.32 & $\pm 0.04 \mathrm{ab}$ & 1.34 & $\pm 0.02 \mathrm{ab}$ & 1.38 & $\pm 0.03 \mathbf{b}$ & 0.05 \\
\hline Arena (\%) & 79.71 & \pm 3.40 & 83.29 & \pm 0.94 & 79.43 & \pm 3.39 & 80.29 & \pm 2.61 & 0.64 \\
\hline Limo (\%) & 6.43 & \pm 0.95 & 6.29 & \pm 0.36 & 6.43 & \pm 1.13 & 7.00 & \pm 0.82 & 0.69 \\
\hline Arcilla (\%) & 13.86 & \pm 2.49 & 10.43 & \pm 1.02 & 14.14 & \pm 2.45 & 12.71 & \pm 2.11 & 0.32 \\
\hline \multicolumn{10}{|l|}{$20-50 \mathrm{~cm}$} \\
\hline $\mathrm{pH}$ & 5.99 & \pm 0.42 & 5.47 & \pm 0.32 & 6.00 & \pm 0.42 & 5.86 & \pm 0.39 & 0.74 \\
\hline CO (\%) & 0.29 & \pm 0.05 & 0.29 & \pm 0.06 & 0.33 & \pm 0.06 & 0.38 & \pm 0.06 & 0.65 \\
\hline MO (\%) & 0.50 & \pm 0.08 & 0.50 & \pm 0.10 & 0.58 & \pm 0.11 & 0.66 & \pm 0.10 & 0.65 \\
\hline $\operatorname{COS}(\mathrm{Mg} / \mathrm{ha})$ & 11.33 & \pm 1.79 & 11.61 & \pm 2.10 & 13.54 & \pm 2.29 & 15.33 & \pm 2.19 & 0.48 \\
\hline $\mathrm{P}(\mathrm{mg} / \mathrm{kg})$ & 1.71 & \pm 0.50 & 1.46 & \pm 0.45 & 1.19 & \pm 0.46 & 1.50 & \pm 0.33 & 0.78 \\
\hline
\end{tabular}




\section{Revista de CIENCIAS AMBIENTALES Tropical Journal of Environmental Sciences}

Revista de Ciencias Ambientales (Trop J Environ Sci) e-ISSN: 2215-3896

(Julio-Diciembre, 2021) . Vol 55(2): 19-44 DOI: https://doi.org/10.15359/rca.55-2.2 Open Access: www.revistas.una.ac.cr/ambientales e-mail: revista.ambientales@una.ac.cr Kubota V., Pulleman M., Domínguez A., Montiel C., Pineda Y., Ortiz A., Kubsch N., Salas D. y Galeano P.

\begin{tabular}{|c|c|c|c|c|c|c|c|c|c|}
\hline \multirow[b]{2}{*}{$\mathrm{Ca}^{+2}(\mathrm{cmolc} / \mathrm{kg})$} & \multicolumn{2}{|c|}{ B } & \multicolumn{2}{|c|}{$\mathbf{Y}$} & \multicolumn{2}{|c|}{$\mathbf{M}$} & \multicolumn{2}{|c|}{$\mathbf{P}$} & \multirow{2}{*}{$\begin{array}{c}\text { Valor } \mathbf{p} \\
0.48\end{array}$} \\
\hline & 1.23 & \pm 0.15 & 1.03 & \pm 0.12 & 1.34 & \pm 0.17 & 1.26 & \pm 0.17 & \\
\hline $\mathrm{Mg}^{+2}(\mathrm{cmolc} / \mathrm{kg})$ & 0.46 & \pm 0.07 & 0.35 & \pm 0.06 & 0.43 & \pm 0.08 & 0.39 & \pm 0.09 & 0.64 \\
\hline $\mathrm{K}^{+}(\mathrm{cmolc} / \mathrm{kg})$ & 0.13 & \pm 0.04 & 0.18 & \pm 0.09 & 0.08 & \pm 0.02 & 0.12 & \pm 0.02 & 0.57 \\
\hline $\mathrm{Na}^{+}(\mathrm{cmolc} / \mathrm{kg})$ & 0.04 & \pm 0.01 & 0.04 & \pm 0.01 & 0.04 & \pm 0.01 & 0.04 & \pm 0.01 & 1.00 \\
\hline $\mathrm{Al}^{+3}+\mathrm{H}^{+}(\mathrm{cmolc} / \mathrm{kg})$ & 0.31 & \pm 0.22 & 0.58 & \pm 0.26 & 0.40 & \pm 0.28 & 0.54 & \pm 0.25 & 0.85 \\
\hline $\mathrm{N}(\%)$ & 0.02 & \pm 0.00 & 0.01 & \pm 0.00 & 0.01 & \pm 0.00 & 0.02 & \pm 0.00 & 0.47 \\
\hline $\mathrm{CIC}(\mathrm{cmolc} / \mathrm{kg})$ & 2.37 & \pm 0.34 & 2.17 & \pm 0.23 & 2.57 & \pm 0.47 & 2.14 & \pm 0.29 & 0.93 \\
\hline $\mathrm{DA}\left(\mathrm{Mg} / \mathrm{m}^{3}\right)$ & 1.30 & \pm 0.01 & 1.35 & \pm 0.02 & 1.37 & \pm 0.03 & 1.35 & \pm 0.01 & 0.08 \\
\hline Arena (\%) & 81.71 & \pm 4.04 & 84.57 & \pm 1.67 & 78.86 & \pm 4.31 & 80.86 & \pm 2.71 & 0.74 \\
\hline Limo (\%) & 5.43 & \pm 1.23 & 5.00 & \pm 0.72 & 6.71 & \pm 1.21 & 6.29 & \pm 0.84 & 0.61 \\
\hline Arcilla (\%) & 12.86 & \pm 3.22 & 10.43 & \pm 1.85 & 14.43 & \pm 3.17 & 12.86 & \pm 2.14 & 0.76 \\
\hline
\end{tabular}

Apéndice 6. Comparación de medias y error estándar de materia orgánica particular (MOP), macroagregado mayor, macroagregado menor e infiltración de suelo de bosques nativos alterados (B), sistema agroforestal con yerba mate $(\mathrm{Y})$, cultivos de subsistencia $(\mathrm{M})$ y pasturas $(\mathrm{P})$.

Appendix 6. Comparison of means and standard error of particulate organic matter (POM), macroaggregates, microaggregates and infiltration of soil of disturbed native forests (B), agroforestry systems with yerba mate $(\mathrm{Y})$, subsistence crops $(\mathrm{M})$ and pastures $(\mathrm{P})$.

\begin{tabular}{lcccc}
\hline & B & Y & M & P \\
\hline MOP ( $\geq 2 \mathrm{~mm}, \%)$ & $7.44 \pm 2.08 \mathbf{~ a b}$ & $4.60 \pm 0.66 \mathbf{a b}$ & $3.24 \pm 1.50 \mathbf{b}$ & $9.64 \pm 1.48 \mathbf{a}$ \\
Macros ( $\geq 250 \mathrm{um}, \%)$ & $47.50 \pm 2.28$ & $46.68 \pm 3.43$ & $45.52 \pm 2.74$ & $39.24 \pm 3.39$ \\
Micros (53-250 um, \%) & $45.06 \pm 3.89$ & $48.72 \pm 3.17$ & $51.23 \pm 3.88$ & $51.12 \pm 4.08$ \\
Infiltración (segundos) & $14.12 \pm 2.79$ & $12.29 \pm 2.21$ & $9.65 \pm 1.42$ & 0.02 \\
\hline
\end{tabular}

\begin{tabular}{|c|c|c|}
\hline 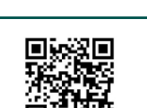 & (ब)(0) & 6 \\
\hline
\end{tabular}

